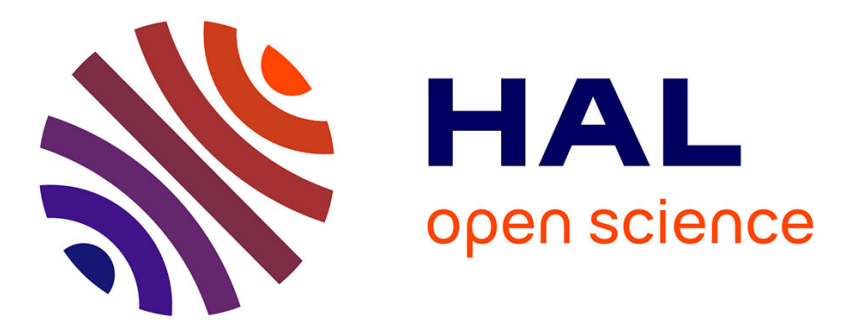

\title{
Fast and slow effective waves across dilute random distributions of elastic spheres in a poroelastic medium
}

Adjovi Kuagbenu, Hervé Franklin, Amah Séna D’almeida

\section{To cite this version:}

Adjovi Kuagbenu, Hervé Franklin, Amah Séna D'almeida. Fast and slow effective waves across dilute random distributions of elastic spheres in a poroelastic medium. Ultrasonics, 2021, 115, pp.106432. 10.1016/j.ultras.2021.106432 . hal-03268373

\section{HAL Id: hal-03268373}

\section{https://hal-normandie-univ.archives-ouvertes.fr/hal-03268373}

Submitted on 7 Sep 2021

HAL is a multi-disciplinary open access archive for the deposit and dissemination of scientific research documents, whether they are published or not. The documents may come from teaching and research institutions in France or abroad, or from public or private research centers.
L'archive ouverte pluridisciplinaire HAL, est destinée au dépôt et à la diffusion de documents scientifiques de niveau recherche, publiés ou non, émanant des établissements d'enseignement et de recherche français ou étrangers, des laboratoires publics ou privés. 


\title{
Fast and slow effective waves across dilute random distributions of elastic spheres in a poroelastic medium
}

\author{
Adjovi Kuagbenu ${ }^{1}$, Hervé Franklin ${ }^{1, a}$ and Amah Séna d'Almeida ${ }^{2}$ \\ ${ }^{1}$ Laboratoire Ondes et Milieux Complexes (LOMC) UMR CNRS 6294, 75 rue Bellot, \\ Université Le Havre Normandie, Le Havre, France \\ ${ }^{2}$ Département de Mathématiques, Université de Lomé, Togo \\ ${ }^{a}$ corresponding author : herve.franklin@univ-lehavre.fr
}

\section{Abstract}

A dilute random distribution of identical elastic spheres in a poroelastic isotropic matrix obeying Biot's theory is considered. Using Luppe, Conoir and Norris (LCN) multiple scattering formula up to the corrective second order term in concentration, approximations are sought in the low frequency domain (Rayleigh limit) for the fast and slow effective wavenumbers. The contribution of the corrective second order term - which contains the coupling (i.e. mode conversions) between the fast, slow and shear waves and accounts for multiple scattering - is discussed. Considering the fast and slow wavenumbers, some effective quantities (bulk modulus, mass density and diffusion coefficient) are estimated.

Keywords: Poroelastic matrix, Random distribution of spheres, Scattering, Low frequency, Bulk modulus, Diffusion coefficient.

\section{Introduction}

Important advances have been made in the understanding of dynamic 
properties of composite materials. In particular, the particulate composites generally man-made - are particles of one type material embedded in a matrix of another. While the case of elastic matrices has received great attention, the same is not true for matrices having more complex properties at low frequency such as poroelastic media. Composite materials whose matrix is poroelastic can exhibit a certain number of properties which can be of interest and which we will examine here.

A heterogeneous medium made of a random distribution of identical elastic spheres in a poroelastic matrix saturated by a viscous liquid is then considered. The poroelastic medium obeys Biot's theory [1] [2], [3] and the radius of the spherical inclusions is assumed far greater than that of the mean pore radius. According to Biot's theory, three dispersive and attenuated plane waves - fast, slow and transverse (shear), the two first being of longitudinal type - exist in an infinite poroelastic medium free of scatterers. Fast and shear waves are propagative regardless of frequency. Slow waves are diffusive at low frequencies, only becoming propagative as the frequency increases.

When each of these waves hits a sphere, the latter scatters fast, slow and transverse waves. This problem of scattering by a single obstacle in a poroelastic medium has been studied by several authors. Using the transition matrix method [10], Kargl and Lim [5] presented general results (extinction, scattering and absorption cross-sections) for porous scatterers of any shape. Zimmerman and Stern [6] examined the problem of fast and slow wave scattering by a spherical inclusion (fluid or elastic), while Berryman [7] performed a multipole expansion of the scattered field by a spherical inhomogeneity excited by fast waves. 
During an incidence on the heterogeneous medium each of these waves undergoes multiple scattering and by an elaborate averaging process gives rise to an effective (coherent) wave of the same type than the incoming wave. Thereby, there are two effective longitudinal waves (fast and slow) and one shear wave assumed all to be plane waves evolving in a homogeneous continuous medium -the effective medium. The associated wavenumbers - the effective wavenumbers - depend on both the concentration of spheres and on the properties of the involved materials, causing the effective medium to remain dispersive like the porous medium free of scatterers, however with its own specificities.

The effects of the multiple scattering on the propagation of the coherent waves - with host media sustaining either one wave (case of perfect fluids), two waves (case of elastic solids) or three waves (case of poroelastic materials) raised a lot of questions in the past. Attempts to answer them led to famous formulas for the coherent wavenumbers and to the issue of obtaining the physical parameters of effective media. Scattering and multiple scattering have been studied extensively over the past 75 years (i) in perfect fluids [8], [9], [10], [11], [12], [13], [14], [15], [16], [17], [18], [19], [20], (ii) in elastic matrix sustaining two waves ; see for example the papers by Kuster and Toksöz [21] on propagation of seismic waves in two phase media, Norris [22] on composites containing dilute concentration of spherical inclusions, Varadan et al. [23] on fiber reinforced composite materials and Aristégui and Angel [24] on composites of cylinders or spheres and the book by Christensen [25]. (iii) in thermo-visco-elastic liquids sustaining three waves (compressional, shear and thermal) ; see for example Epstein and Cahart [26], Allegra and 
Cawley [27]. These authors proposed the ECAH formulation.

Among the most famous formulas for expressing the coherent wavenumbers are those of the ISA (Independent Scattering approximation) [8] and of Waterman and Truell (WT) [10], both derived from the QCA (Quasi Cristalline Approximation) of Lax [9]. An application of the ISA when using an equivalent fluid model to describe the acoustic propagation in the host porous medium (air saturated polymer foam) with parallel rigid cylinders as scatterers was made by Tournat et al. [28]. From 2005, Linton and Martin (LM) revisited and applied to acoustics (considering distributions of spheres [16] and of cylinders [17] in a fluid) the Lloyd and Berry [12] formula. Their works constitute an improvement of WT's [10] formula in the case of low concentrations. Extensions of LM works are avalaible in the case of cylinders in an elastic matrix [29] and spheres in a thermo-visco-elastic medium [30] the latter, referred to as the LCN model being an extension of the ECAH formulation to account for higher order multiple scattering events.

Our objective is to identify, from the propagation of fast and slow waves across the random medium described above, some effective properties at low frequency. Based on the work of Ref. [30], we present briefly in sec. 2 the multiple scattering model in the case of a poroelastic host matrix and the general formulas for the fast and slow effective wavenumbers. These formulas needing the scattering coefficients by one sphere. Sec. 3 is therefore devoted to the calculation of these coefficients and to the low frequency expansions in dimensionless wavenumbers. These approximations form the basis which will subsequently make it possible, via additional expansions, to determine the effective quantities from the effective wavenumbers. In 
Sec. 4, a numerical study considering aluminum spheres in QF20@ a watersaturated porous material described in Ref. [31] - allow us to check the validity of the first set of low frequency approximations obtained in sec. 3 . The fast and slow effective wavenumbers are computed and the resulting velocities and attenuations compared with those of the poroelastic medium free of scatterers. In sec. 5, explicit low frequency expressions supplementing the low frequency approximations in dimensionless wavenumbers of sec. 3 are considered to further simplify the formulas of the wavenumbers. This allows, to extract in sec. 6 the static effective bulk modulus, mass density as a consequence of the fast wave and in sec. 7 the effective diffusion coefficient as a consequence of the slow wave.

\section{The multiple scattering model}

\subsection{Far-field scattered amplitudes for a sphere}

Consider an elastic sphere of radius $R$ centred at the origin of the axes and set in a fluid-saturated poroelastic matrix. The physical properties of the poroelastic matrix are listed in Table 1 . The mean pore radius $a_{p}$ is such that $a_{p} \ll R\left(=4 \times 10^{-3}\right)$. The elastic sphere has Lamé coefficients $\lambda_{s}, \mu_{s}$ and mass density $\rho_{s}\left(c_{L}=\sqrt{\left(\lambda_{s}+2 \mu_{s}\right) / \rho_{s}}\right.$ and $c_{T}=\sqrt{\mu_{s} / \rho_{s}}$ denote longitudinal and transverse velocities, see values used in Table 2). When a plane harmonic wave of type $\alpha(=1$ or 2$)$ - time dependance of the form $e^{-i \omega \tau}$ with $\omega$ the angular frequency and $\tau$ the time - hits the sphere, it gives rise to scattered spherical waves of type $\beta(=1,2$ (longitudinal) or $t$ (transverse)). 
The far-field scattered amplitudes of a sphere are given by

$$
f^{\alpha \beta}(\theta)=\sum_{n=0}^{+\infty}(2 n+1) t_{n}^{\alpha \beta} \mathrm{P}_{n}(\cos \theta)
$$

if $\alpha=1,2$ and

$$
f^{t \beta}(\theta, \phi)=\sum_{n=1}^{+\infty} \frac{(2 n+1)}{n(n+1)} t_{n}^{t \beta} \mathrm{P}_{n}^{1}(\cos \theta) \cos \phi
$$

if $\alpha=t$; see Brill and Gaunaurd [36] in the case of a sphere in an elastic matrix. Above, $t_{n}^{\alpha \beta}$ denotes the scattering coefficient for the mode $n$. It is calculated by solving the linear system derived from the open pore boundary conditions, see Eq. (A.1); $\theta$ is the polar angle, $\phi$ the azimuthal angle, $\mathrm{P}_{n}(\cos \theta)$ the Legendre polynomial of index $n$ and $\mathrm{P}_{n}^{1}(\cos \theta)=\sin \theta \times d \mathrm{P}_{n}(\cos \theta) / d(\cos \theta)$ the associated Legendre function (of the first kind) [37]. Equation (2) shows

that none of the coefficients $t_{0}^{t \beta}$ is involved in the scattering of a transverse wave by a sphere.

\subsection{Multiple scattering equations}

In LCN's formula of multiple scattering for randomly distributed spheres in themo-visco-elastic fluids [30], the square of the effective wavenumbers $\zeta_{\alpha}$ is given by

$$
\zeta_{\alpha}^{2}=k_{\alpha}^{2}+n_{0} \delta_{1}^{\alpha}+n_{0}^{2}\left(\delta_{2}^{\alpha, 0}+\delta_{2}^{\alpha, c}\right)+O\left(n_{0}^{3}\right),
$$

where $k_{\alpha}$ is the wavenumber of the incident plane wave ( $\alpha=1$ or 2$)$. These expansions with respect to $n_{0}$-the number of spheres per unit volume- assume low concentrations of scatterers, so that terms of order $n_{0}^{3}$ (and the followings) 
accounting for higher orders of interactions between the spheres, can be left out. In Eq. (3),

$$
\begin{gathered}
\delta_{1}^{\alpha}=\frac{4 \pi}{i k_{\alpha}} \sum_{n=0}^{+\infty}(2 n+1) t_{n}^{\alpha \alpha}, \\
\delta_{2}^{\alpha, 0}=-\frac{1}{2}\left(\frac{4 \pi}{k_{\alpha}}\right)^{4} \sum_{n, m=0}^{+\infty} K_{n m} t_{n}^{\alpha \alpha} t_{m}^{\alpha \alpha}, \\
\delta_{2}^{\alpha, c}=-\frac{1}{2}\left(\frac{4 \pi}{k_{\alpha}}\right)^{4} \sum_{n=0, m=0}^{+\infty} \sum_{\beta \neq \alpha} L^{\alpha \beta} K_{n m}^{\alpha \beta} t_{n}^{\beta \alpha} t_{m}^{\alpha \beta},
\end{gathered}
$$

where

$$
\begin{aligned}
L^{\alpha \beta} & =\frac{2 k_{\alpha}^{3}}{k_{\beta}\left(k_{\alpha}^{2}-k_{\beta}^{2}\right)}, \\
K_{n m} & =\left(\frac{1}{4 \pi}\right)^{\frac{3}{2}} \sqrt{(2 n+1)(2 m+1)} \sum_{q} q \sqrt{2 q+1} G(n, 0 ; m, 0 ; q),
\end{aligned}
$$

and

$$
K_{n m}^{\alpha \beta}=\left(\frac{1}{4 \pi}\right)^{\frac{3}{2}} \sqrt{(2 n+1)(2 m+1)} \sum_{q}\left(\frac{k_{\alpha}}{k_{\beta}}\right)^{q} \sqrt{2 q+1} G(n, 0 ; m, 0 ; q) .
$$

Above, $G(n, 0 ; m, 0 ; q)$ represents Gaunt's function [38] and the summation on $q$ runs from $|n-m|$ to $n+m$ in steps of two, with $m+n+q$ even. The presenting of Eqs. (5) and (6) differs from Refs [30] and [40]. The calculation of the first coefficients $K_{n m}$ and $K_{n m}^{\alpha \beta}$ yields

$$
\begin{gathered}
K_{00}=0, \quad K_{01}=K_{10}=\frac{3}{16 \pi^{2}}, \quad K_{11}=\frac{3}{4 \pi^{2}}, \\
K_{00}^{\alpha \beta}=\frac{1}{16 \pi^{2}}, \quad K_{01}^{\alpha \beta}=\frac{3}{16 \pi^{2}} \frac{k_{\alpha}}{k_{\beta}}\left(\equiv K_{10}^{\alpha \beta}\right), \\
K_{11}^{\alpha \beta}=\frac{3}{16 \pi^{2}}\left[1+2\left(\frac{k_{\alpha}}{k_{\beta}}\right)^{2}\right] .
\end{gathered}
$$

By expanding $\delta_{2}^{\alpha, c}$, one can note the presence of scattering coefficients such as $t_{n}^{\alpha t}$ and $t_{n}^{t \alpha}$ due to $t$ waves generated from spheres to spheres during the multiple scattering process. 


\section{Low frequency expansions in dimensionless wavenumbers for the scattering coefficients $t_{n}^{\alpha \beta}$}

In this section, the parameters that characterize wave propagation in the poroelastic medium - namely $\frac{k_{\alpha}}{\omega}, \gamma_{\alpha}$ - are assumed constant (see definition in the Appendix). This allows to perform the Taylor series expansions of the Bessel and Hankel functions about $\omega=0$, that is to say, for dimensionless wavenumbers $\left|x_{\alpha}\right|=\left|k_{\alpha} R\right| \ll 1(\alpha=1,2, t, L, T)$. All $k_{\alpha} R$ products are small for all modes. The calculations show that $t_{0}^{\alpha \beta}=x_{\alpha}^{p} x_{\beta}^{q}(\ldots)+O\left(x^{5}\right)(\alpha=1,2)$, $t_{0}^{\alpha t}=-1(\alpha=1,2, t), t_{0}^{t \beta}=0(\beta=1,2), t_{1}^{\alpha \beta}=x_{\alpha}^{p} x_{\beta}^{q}(\ldots)+O\left(x^{5}\right)(\alpha, \beta=1,2, t)$. The integers $p$ and $q$ are such that $p+q=3$. For $n \geq 2, t_{n}^{\alpha \beta}$ is $O\left(x^{5}\right)$. Thence, the contribution of the modes $n \geq 2$ are neglected from now on. The notation $x^{5}$ means any monomial constituted with products of $x_{\alpha}(\alpha=1,2, t, T, L)$ and reaching a power of five such as for exemple, $x_{1} x_{2} x_{t} x_{T} x_{L}$ or $x_{1}^{3} x_{T}^{2}, \ldots$ etc. The signs (...) denote quantities independent of the radius of the spheres. Explicitly,

$$
\begin{gathered}
t_{0}^{11}=\frac{i x_{1}^{3}}{3}\left(\frac{b_{0}^{11}}{d_{0}}-1\right), \quad b_{0}^{11}=-6 \gamma_{2} c, \\
t_{0}^{12}=\frac{i x_{1}^{2} x_{2}}{3} \frac{b_{0}^{12}}{d_{0}}, \quad b_{0}^{12}=6 \gamma_{1} c, \\
t_{0}^{22}=\frac{i x_{2}^{3}}{3}\left(\frac{b_{0}^{22}}{d_{0}}-1\right), \quad b_{0}^{22}=6 \gamma_{1} b, \\
t_{0}^{21}=-\frac{i x_{1} x_{2}^{2}}{3} \frac{b_{0}^{21}}{d_{0}}, \quad b_{0}^{21}=6 \gamma_{2} b, \\
d_{0}=-2 \gamma_{21}\left[3 \lambda_{s}\left(\mu-\mu_{s}\right)+4 \mu^{2}-2 \mu_{s}\left(\mu+\mu_{s}\right)\right],
\end{gathered}
$$

for the mode $n=0$ and

$$
t_{1}^{11}=\frac{-i x_{1}^{3}}{3} \frac{b_{1}^{11}}{d_{1}}, \quad t_{1}^{12}=\frac{-i x_{1} x_{2}^{2}}{3} \frac{b_{1}^{12}}{d_{1}}, \quad t_{1}^{1 t}=\frac{-i x_{1} x_{t}^{2}}{3} \frac{b_{1}^{1 t}}{d_{1}},
$$




$$
\begin{aligned}
t_{1}^{21}=\frac{-i x_{2} x_{1}^{2}}{3} \frac{b_{1}^{21}}{d_{1}}, & t_{1}^{22}=\frac{-i x_{2}^{3}}{3} \frac{b_{1}^{22}}{d_{1}}, & t_{1}^{2 t}=\frac{-i x_{2} x_{t}^{2}}{3} \frac{b_{1}^{2 t}}{d_{1}}, \\
t_{1}^{t 1}=\frac{-i x_{1}^{2} x_{t}}{3} \frac{b_{1}^{t 1}}{d_{1}}, & t_{1}^{t 2}=\frac{-i x_{2}^{2} x_{t}}{3} \frac{b_{1}^{t 2}}{d_{1}}, & t_{1}^{t t}=\frac{-i x_{t}^{3}}{3} \frac{b_{1}^{t t}}{d_{1}}
\end{aligned}
$$

for the mode $n=1$, where

$$
\begin{aligned}
& d_{1}= 4 \gamma_{21} a \frac{x_{t}^{2}}{x_{T}^{2}}+8 \gamma_{t 1} b \frac{x_{2}^{2}}{x_{T}^{2}}+8 \gamma_{2 t} c \frac{x_{1}^{2}}{x_{T}^{2}}+8 \gamma_{21} f \frac{x_{t}^{2}}{x_{L}^{2}}+2 \gamma_{t 1} d \frac{x_{2}^{2}}{x_{L}^{2}}+2 \gamma_{2 t} e \frac{x_{1}^{2}}{x_{L}^{2}} \\
& b_{1}^{11}= 2 \gamma_{1} a \frac{x_{t}^{2}}{x_{T}^{2}}+4 \gamma_{1} b \frac{x_{2}^{2}}{x_{T}^{2}}+8 \gamma_{2 t} c \frac{x_{1}^{2}}{x_{T}^{2}}+4 \gamma_{1} f \frac{x_{t}^{2}}{x_{L}^{2}}+\gamma_{1} d \frac{x_{2}^{2}}{x_{L}^{2}}+2 \gamma_{2 t} e \frac{x_{1}^{2}}{x_{L}^{2}}-2 \gamma_{2 t} h \\
& b_{1}^{12}=-2 \gamma_{1} a \frac{x_{t}^{2}}{x_{T}^{2}}+4\left(2 \gamma_{t}-3 \gamma_{1}\right) c \frac{x_{1}^{2}}{x_{T}^{2}}-4 \gamma_{1} f \frac{x_{t}^{2}}{x_{L}^{2}}+\left(2 \gamma_{t}-3 \gamma_{1}\right) e \frac{x_{1}^{2}}{x_{L}^{2}}-2 \gamma_{t 1} h, \\
& b_{1}^{1 t}= 4 \gamma_{1} b \frac{x_{2}^{2}}{x_{T}^{2}}+4\left(2 \gamma_{2}-3 \gamma_{1}\right) c \frac{x_{1}^{2}}{x_{T}^{2}}+\gamma_{1} d \frac{x_{2}^{2}}{x_{L}^{2}}+\left(2 \gamma_{2}-3 \gamma_{1}\right) e \frac{x_{1}^{2}}{x_{L}^{2}}-2 \gamma_{21} h, \\
& b_{1}^{21}= 2 \gamma_{2} a \frac{x_{t}^{2}}{x_{T}^{2}}-4\left(2 \gamma_{t}-3 \gamma_{2}\right) b \frac{x_{2}^{2}}{x_{T}^{2}}+4 \gamma_{2} f \frac{x_{t}^{2}}{x_{L}^{2}}-\left(2 \gamma_{t}-3 \gamma_{2}\right) d \frac{x_{2}^{2}}{x_{L}^{2}}-2 \gamma_{2 t} h, \\
& b_{1}^{22}=-2 \gamma_{2} a \frac{x_{t}^{2}}{x_{T}^{2}}-4 \gamma_{2} c \frac{x_{1}^{2}}{x_{T}^{2}}+8 \gamma_{t 1} b \frac{x_{2}^{2}}{x_{T}^{2}}-4 \gamma_{2} f \frac{x_{t}^{2}}{x_{L}^{2}}-\gamma_{2} e \frac{x_{1}^{2}}{x_{L}^{2}}+2 \gamma_{t 1} d \frac{x_{2}^{2}}{x_{L}^{2}}-2 \gamma_{t 1} h, \\
& b_{1}^{2 t}=-4 \gamma_{2} c \frac{x_{1}^{2}}{x_{T}^{2}}+4\left(3 \gamma_{2}-2 \gamma_{1}\right) b \frac{x_{2}^{2}}{x_{T}^{2}}-\gamma_{2} e \frac{x_{1}^{2}}{x_{L}^{2}}+\left(3 \gamma_{2}-2 \gamma_{1}\right) d \frac{x_{2}^{2}}{x_{L}^{2}}-2 \gamma_{21} h \\
& b_{1}^{t 1}=8 \gamma_{t} b \frac{x_{2}^{2}}{x_{T}^{2}}+4 \gamma_{2} a \frac{x_{t}^{2}}{x_{T}^{2}}+\gamma_{t} d \frac{x_{2}^{2}}{x_{L}^{2}}+8 \gamma_{2} f \frac{x_{t}^{2}}{x_{L}^{2}}-4 \gamma_{2 t} h \\
& b_{1}^{t 2}=-8 \gamma_{t} c \frac{x_{1}^{2}}{x_{T}^{2}}-4 \gamma_{1} a \frac{x_{t}^{2}}{x_{T}^{2}}-\gamma_{t} e \frac{x_{1}^{2}}{x_{L}^{2}}-8 \gamma_{1} f \frac{x_{t}^{2}}{x_{L}^{2}}-4 \gamma_{t 1} h \\
&\left.x_{T}^{2}-c \frac{x_{1}^{2}}{x_{T}^{2}}\right)+4 \gamma_{21} a \frac{x_{t}^{2}}{x_{T}^{2}}+2 \gamma_{t}\left(d \frac{x_{2}^{2}}{x_{L}^{2}}-e \frac{x_{1}^{2}}{x_{L}^{2}}\right)+8 \gamma_{21} f \frac{x_{t}^{2}}{x_{L}^{2}}-4 \gamma_{21} h
\end{aligned}
$$

The following abbreviations were used

$$
\begin{aligned}
& a=\mu\left(5 \lambda_{s}+4 \mu+6 \mu_{s}\right), \\
& b=\left(\mathcal{H}_{2}+2 \mu\right)\left(\mu-\mu_{s}\right),
\end{aligned}
$$




$$
\begin{gathered}
c=\left(\mathcal{H}_{1}+2 \mu\right)\left(\mu-\mu_{s}\right), \\
d=\left(\mathcal{H}_{2}+2 \mu\right)\left(2 \mu+3 \mu_{s}\right), \\
e=\left(\mathcal{H}_{1}+2 \mu\right)\left(2 \mu+3 \mu_{s}\right), \\
f=\mu\left(\mu-\mu_{s}\right), \\
h=\lambda_{s}\left(2 \mu+3 \mu_{s}\right)+2 \mu_{s}\left(4 \mu+\mu_{s}\right),
\end{gathered}
$$

and

$$
\gamma_{21}=\gamma_{2}-\gamma_{1}, \quad \gamma_{t 1}=\gamma_{t}-\gamma_{1}, \quad \gamma_{2 t}=\gamma_{2}-\gamma_{t}
$$

The moduli $\mu, \mathcal{H}_{j}\left(j=1,2\right.$, Eq. (A.32)), the dimensionless factors $\gamma_{j}(j=$ 1, 2, Eq. (A.30)) and $\gamma_{t}$ (Eq. (A.31)) were defined in the Appendix. The magnitudes of the scattering coefficients $t_{0}^{\alpha t}$ are given here for information, but according to the definition of the scattering cross section by Yin and Truell [39], they should be excluded - see also the discussion in Ref. [40].

\subsection{Formulas for the fast effective wavenumber}

Since we are only interested in terms up to $O\left(x^{3}\right)$ in the scattering coefficients, the series Eqs. $(4-6)$ can be truncated to contain $t_{0}^{\alpha \beta}$ and $t_{1}^{\alpha \beta}$ only. Substituting for the approximations of $t_{n}^{\alpha \beta}$, Eqs. (13-16) and Eqs. (18-20), in the formulas for $\delta_{1}^{1}, \delta_{2}^{1,0}$ and $\delta_{2}^{1, c}$, we obtain after some rearrangements

$$
\begin{aligned}
\frac{n_{0}}{k_{1}^{2}} \delta_{1}^{1}= & \mathfrak{C}\left[\frac{b_{0}^{11}}{d_{0}}-1-3 \frac{b_{1}^{11}}{d_{1}}\right] \\
\frac{n_{0}^{2}}{k_{1}^{2}} \delta_{2}^{1,0}= & \mathfrak{C}^{2}\left[3\left(1-\frac{b_{0}^{11}}{d_{0}}\right) \frac{b_{1}^{11}}{d_{1}}+6\left(\frac{b_{1}^{11}}{d_{1}}\right)^{2}\right] \\
\frac{n_{0}^{2}}{k_{1}^{2}} \delta_{2}^{1, c}= & -\frac{1}{2} \mathfrak{C}^{2} \times\left\{L ^ { 1 2 } \left[\left(\frac{k_{2}}{k_{1}}\right)^{3} \frac{b_{0}^{21} b_{0}^{12}}{d_{0}^{2}}+3 \frac{k_{1}}{k_{2}}\left(-\left(\frac{k_{2}}{k_{1}}\right)^{4} \frac{b_{0}^{21} b_{1}^{12}}{d_{0} d_{1}}+\left(\frac{k_{2}}{k_{1}}\right)^{2} \frac{b_{1}^{21} b_{0}^{12}}{d_{1} d_{0}}\right)\right.\right. \\
& \left.-3\left(1+2\left(\frac{k_{1}}{k_{2}}\right)^{2}\right)\left(\frac{k_{2}}{k_{1}}\right)^{3} \frac{b_{1}^{12} b_{1}^{21}}{d_{1}^{2}}\right]
\end{aligned}
$$




$$
\left.-L^{1 t}\left[\left(1+2\left(\frac{k_{1}}{k_{t}}\right)^{2}\right)\left(\frac{k_{t}}{k_{1}}\right)^{3} \frac{b_{1}^{1 t} b_{1}^{t 1}}{d_{1}^{2}}\right]\right\},
$$

where $\mathfrak{C}=n_{0}\left(4 \pi R^{3} / 3\right)$ is the particle volume fraction. A formula of the effective wavenumber, correct to the first order in $\mathfrak{C}$ (the contributions from $\delta_{2}^{1, c}$ are then ignored), is given as a product of two factors

$$
\frac{\zeta_{1}^{2}}{k_{1}^{2}}=\left[1-3 \mathfrak{C} \frac{b_{1}^{11}}{d_{1}}\right]\left[1+\mathfrak{C}\left(\frac{b_{0}^{11}}{d_{0}}-1\right)\right],
$$

Eq. (42) is a form allowing to extract later effective density and modulus [18]. Note that it contains a term $3 \mathfrak{C}^{2}\left(1-\frac{b_{0}^{11}}{d_{0}}\right) \frac{b_{1}^{11}}{d_{1}}$ present in $\frac{n_{0}^{2}}{k_{1}^{2}} \delta_{2}^{1,0}$ which accounts for mulptiple scattering without mode conversions.

\subsection{Formulas for the slow effective wavenumber}

In the case of an incident slow wave on the random distribution, we obtain the following expressions for the $\delta s$

$$
\begin{aligned}
\frac{n_{0}}{k_{2}^{2}} \delta_{1}^{2}= & \mathfrak{C}\left[\frac{b_{0}^{22}}{d_{0}}-1-3 \frac{b_{1}^{22}}{d_{1}}\right] \\
\frac{n_{0}^{2}}{k_{2}^{2}} \delta_{2}^{2,0}= & \mathfrak{C}^{2}\left[3\left(1-\frac{b_{0}^{22}}{d_{0}}\right) \frac{b_{1}^{22}}{d_{1}}+6\left(\frac{b_{1}^{22}}{d_{1}}\right)^{2}\right] \\
\frac{n_{0}^{2}}{k_{2}^{2}} \delta_{2}^{2, c}= & -\frac{1}{2} \mathfrak{C}^{2}\left\{L ^ { 2 1 } \left[\left(\frac{k_{1}}{k_{2}}\right)^{3} \frac{b_{0}^{12} b_{0}^{21}}{d_{0}^{2}}+3 \frac{k_{2}}{k_{1}}\left(\left(\frac{k_{1}}{k_{2}}\right)^{4} \frac{b_{0}^{12} b_{1}^{21}}{d_{0} d_{1}}-\left(\frac{k_{1}}{k_{2}}\right)^{2} \frac{b_{1}^{12} b_{0}^{21}}{d_{1} d_{0}}\right)\right.\right. \\
& \left.-3\left(1+2\left(\frac{k_{2}}{k_{1}}\right)^{2}\right)\left(\frac{k_{1}}{k_{2}}\right)^{3} \frac{b_{1}^{12} b_{1}^{21}}{d_{1}^{2}}\right] \\
& \left.-L^{2 t}\left[\left(1+2\left(\frac{k_{2}}{k_{t}}\right)^{2}\right)\left(\frac{k_{t}}{k_{2}}\right)^{3} \frac{b_{1}^{2 t} b_{1}^{t 2}}{d_{1}^{2}}\right]\right\}
\end{aligned}
$$

In the same way as above, one could factorize the slow effective wavenumber and write a valid first order concentration formula similar to Eq. (42). The difficulty encountered here is the radically different behavior of the slow wave with respect to the fast wave. Clearly, factorization seems justified for 
extracting two effective quantities from the same formula, that is modulus and mass density. From the effective slow wavenumber it will be possible to extract only one effective quantity (the behavior of the terms $b_{0}^{22}$, Eq. (15), and $b_{1}^{22}$, Eq. (26), at very low frequencies leads to this conclusion, see sec. 7).

\begin{tabular}{|c|c|c|}
\hline Parameters for the poroelastic medium [31] & Units & Values \\
\hline$K_{b}$ bulk modulus of (dry) porous frame & $\mathrm{Pa}$ & $9.47 \times 10^{9}$ \\
\hline $\bar{K}_{s}$ bulk modulus of solid grains & $\mathrm{Pa}$ & $3.66 \times 10^{10}$ \\
\hline$K_{f}$ bulk modulus of water & $\mathrm{Pa}$ & $2.22 \times 10^{9}$ \\
\hline$\mu$ shear modulus of (dry) porous frame & $\mathrm{Pa}$ & $7.63 \times 10^{9}$ \\
\hline$\overline{\rho_{s}}$ mass density of solid grains & $\operatorname{kg~m}{ }^{-3}$ & 2760 \\
\hline$\rho_{f}$ mass density of water (saturating fluid) & $\mathrm{kg} \mathrm{m}^{-3}$ & 1000 \\
\hline$\phi$ porosity & - & 0.402 \\
\hline$\kappa$ permeability & $\mathrm{m}^{2}$ & $1.68 \times 10^{-11}$ \\
\hline$a_{p}$ mean pore radius (or pore size parameter) & $\mathrm{m}$ & $3.26 \times 10^{-5}$ \\
\hline$\eta$ dynamic viscosity & $\mathrm{kg} \mathrm{m}^{-1} \mathrm{~s}^{-1}$ & $1.14 \times 10^{-3}$ \\
\hline$\tau_{0}$ tortuosity & - & 1.89 \\
\hline
\end{tabular}

Table 1: Parameters for the poroelastic matrix - QF20® by Filtros, Ref. [31] - and the saturating fluid (water).

\section{Numerical study}

\subsection{Scattering coefficients}

The data in Tables 1 and 2 were used all along the paper. Additional information regarding the poroelastic medium QF20ß manufactured by 


\begin{tabular}{|l|l|l|l|}
\hline Parameters for elastic spheres & Units & aluminum & epoxy \\
\hline$\rho_{s}$ mass density & $\mathrm{kg} \mathrm{m}^{-3}$ & 2761 & 1180 \\
$c_{L}$ velocity of longitudinal waves & $\mathrm{m} \mathrm{s}^{-1}$ & 6363 & 2540 \\
$c_{T}$ velocity of transverse waves & $\mathrm{m} \mathrm{s}^{-1}$ & 3161 & 1160 \\
\hline
\end{tabular}

Table 2: Parameters for the elastic spheres (aluminum from Ref. [34], epoxy from Ref. $[35])$.

Filtros, can be found in Ref. [31].

The numerical calculations were performed using Matlabß . Figure 1 shows the dimensionless wavenumbers $\left|x_{\alpha}\right|(\alpha=1,2, t)$ vs. the frequency. At frequencies larger than $38 \mathrm{kHz},\left|x_{2}\right|$ exceeds one. As already pointed out in a previous paper, cf. sec. II.B of Ref. [41], it very often happens that in physics predictions drawn from a low frequency theory agree with experimental results obtained at higher frequencies. This is the reason why we go here up to the value $f_{\max }=50 \mathrm{kHz}$ (although, approching this value, the divergences become non negligible between some of the exact formulas of the scattering coefficients and their approximations from Taylor expansions about $\omega=0)$. To fulfill $\left|x_{2}\right|<<1$, it would be necessary to consider only frequencies $f<10 \mathrm{kHz}$, which is very restrictive (note that with $x_{2}$ fixed, spheres smaller than those considered here make it possible to consider higher frequencies).

Figure 2 shows the modulus of the exact and approximate scattering coefficients $t_{0}^{1 \beta}, t_{0}^{2 \beta}, t_{1}^{1 \beta}$ and $t_{1}^{2 \beta}(\beta=1,2)$ vs. the frequency for an aluminum sphere of radius $R=4 \times 10^{-3} \mathrm{~m}$. In the case of the scattering coefficients $t_{0}^{21}$ and $t_{1}^{21}$, the difference between the exact and approximate scattering coeffi- 
cients grows very quickly past $f=30 \mathrm{kHz}$. This discrepancy originates from the handling of the second member of Eq.(A.1). For $t_{0}^{21}$ and $t_{1}^{21}$, errors accumulate in the approximation of the scattering coefficients because of Taylor expansions also performed on Bessel functions of the argument $x_{2}$ lying in the second member $\vec{S}_{n}^{2}$ (for $n=0$ as well as for $n=1$ ). To highlight simply the most influential parameters of the fluid-saturated poroelastic matrix and the elastic spheres, and the way they act, approximations rather than exact formulas are useful.

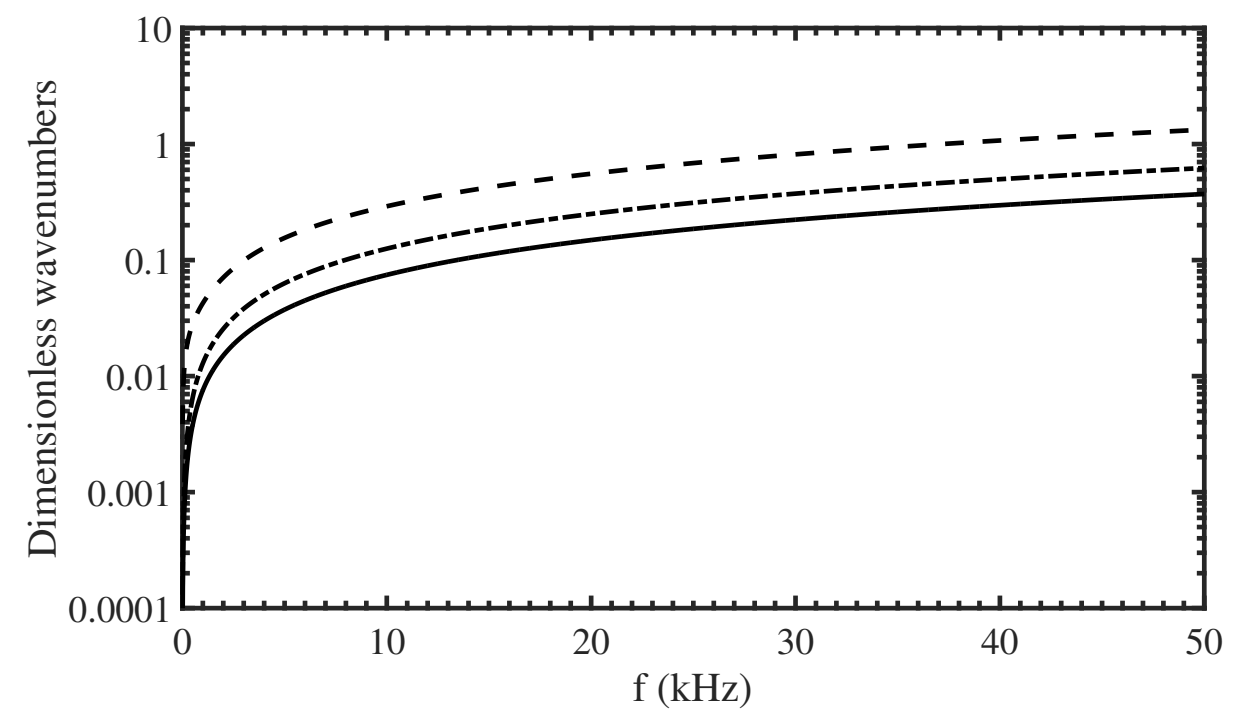

Figure 1: Modulus of the dimensionless wavenumbers $x_{\alpha}=k_{\alpha} R$ (in logarithmic scale) vs. the frequency : $\alpha=1$, fast wave (solid), $\alpha=t$, shear wave (dotdashed) and $\alpha=2$, slow wave (dashed). The radius of the spheres is $R=4 \times 10^{-3} \mathrm{~m}$. 

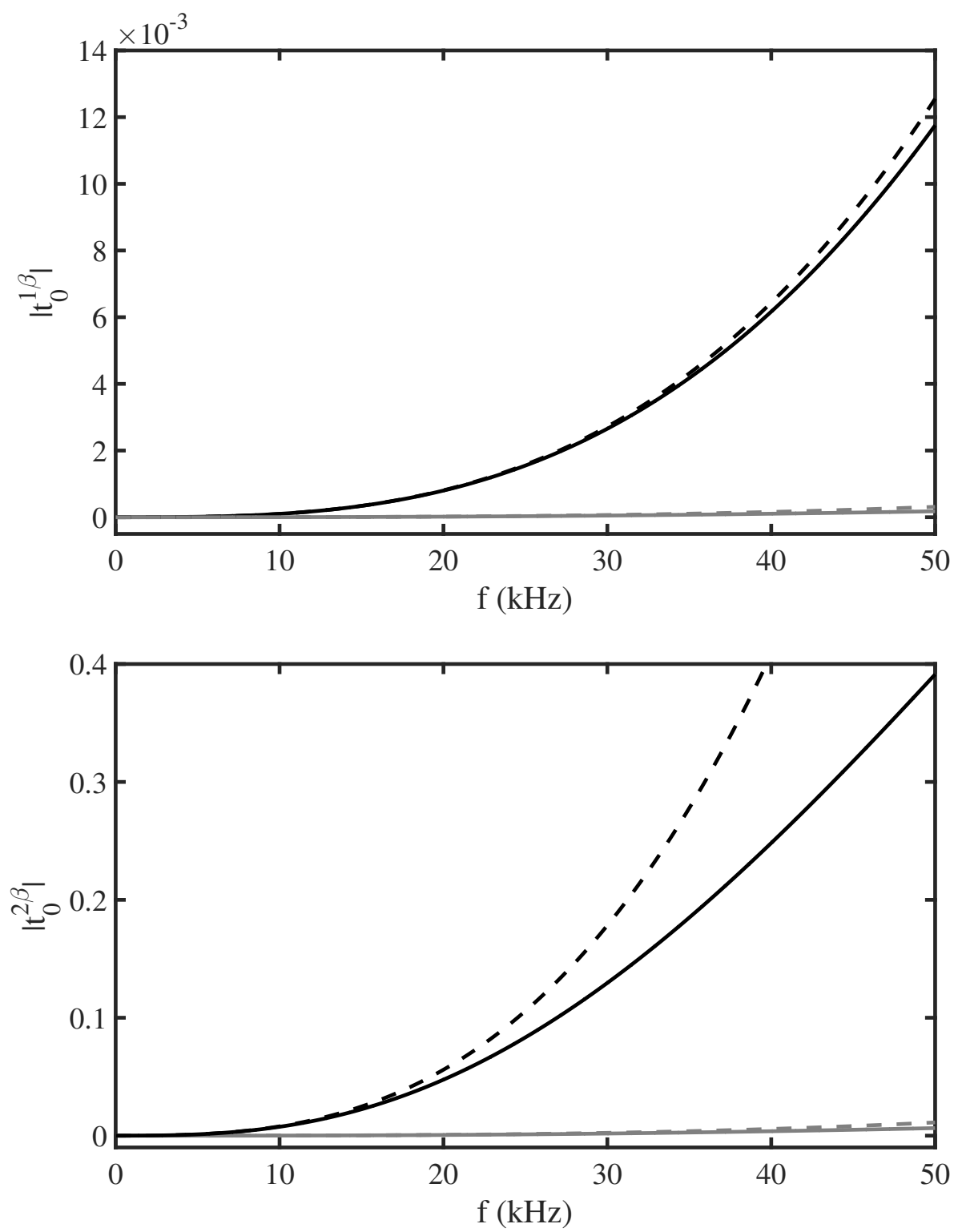

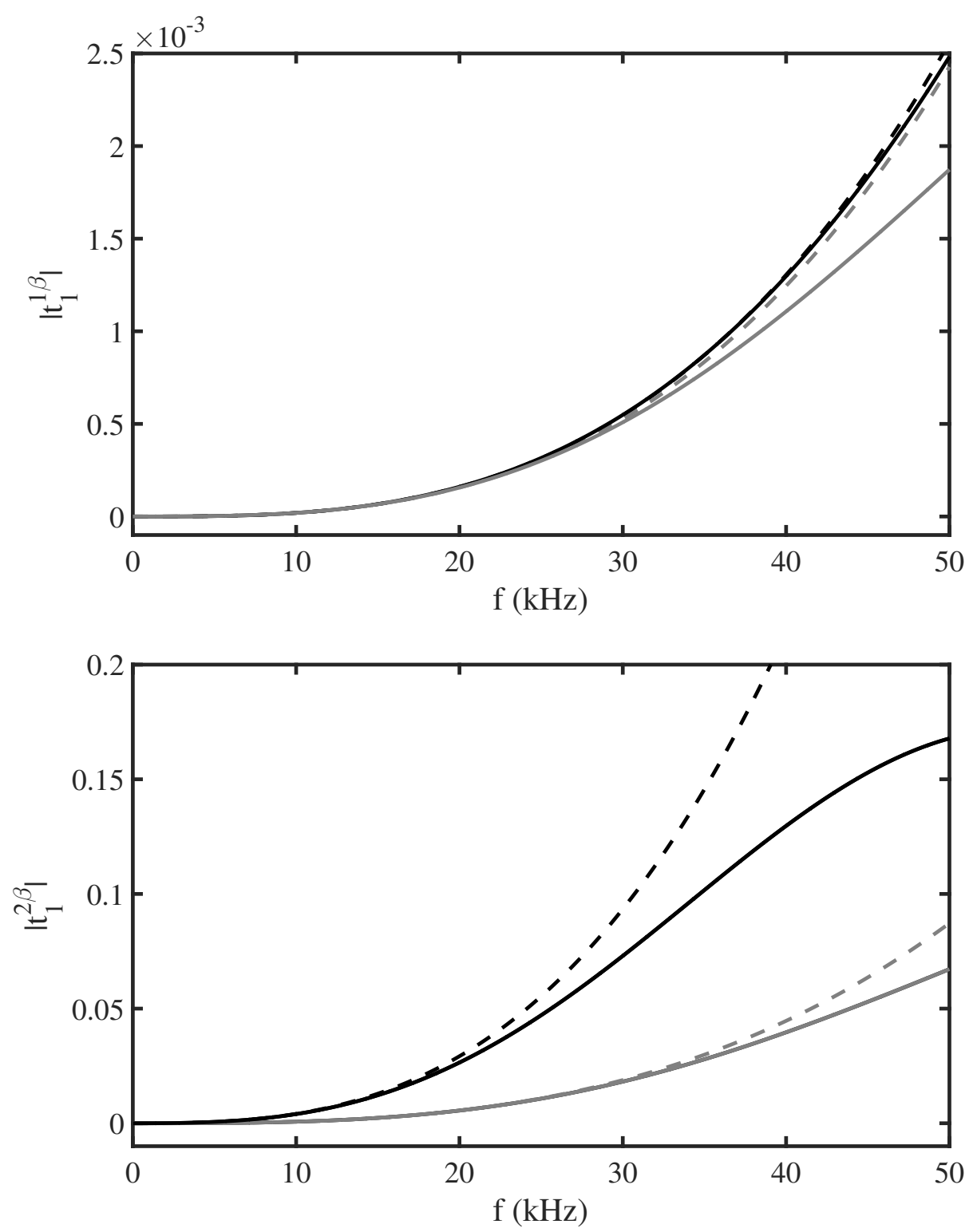

Figure 2: From top to bottom, modulus of the scattering coefficients $t_{0}^{1 \beta}, t_{0}^{2 \beta}, t_{1}^{1 \beta}$ and $t_{1}^{2 \beta}$ vs. the frequency (logarithmic scale). Exact formulas (solid line) and low frequency approximations (dashed line). Black $\beta=1$, gray $\beta=2$. Radius of sphere $R=4 \times 10^{-3} \mathrm{~m}$. 


\subsection{Effective wavenumbers}

\subsubsection{Characteristics of the medium without scatterers}

Velocities $\omega / \operatorname{Re}\left(k_{\alpha}\right)$ and attenuations $\operatorname{Im}\left(k_{\alpha}\right) / \operatorname{Re}\left(k_{\alpha}\right)$ of the fast and slow waves in absence of scatterers are shown in Figs. 3 to 6 (solid curves). Note the low frequency limits for the fast and slow wave velocities : 3313 $\mathrm{m} / \mathrm{s}$ and $0 \mathrm{~m} / \mathrm{s}$; and that of the shear wave (curves not furnished here but the behavior is similar to the fast wave) : $1928 \mathrm{~m} / \mathrm{s}$. The three velocities increase as the frequency goes from 0 to $50 \mathrm{kHz}$. The dispersion is large in the range $0.30-30 \mathrm{kHz}$. Regarding the attenuation, that of the fast wave, Fig. 4, are very moderate compared to the slow wave, Fig. 6, because during their propagation, the motion of the solid frame and saturating fluid is nearly in phase, whereas during the propagation of the slow wave the motion of the fluid in the pores is out of phase with the solid frame. At very low frequency, the slow wave has a non-zero attenuation that characterizes its diffusive nature. Note also that, as the frequency increases from zero to $50 \mathrm{kHz}$, the attenuation of the slow wave decreases from 1 to 0 . The frequency for maximum damping of the fast wave is near $2 \mathrm{kHz}$. It depends on two main factors : the physical parameters of the skeleton and the damping resulting from the friction of the fluid in the pores.

\subsubsection{Effects caused by the scatterers on the waves}

Let us examine now the effect on the fast and slow waves of a random distribution of aluminum spheres (radius $R=4 \times 10^{-3} \mathrm{~m}$, particle volume fraction $\mathfrak{C}=20 \%)$. Results of calculations for $\omega / \operatorname{Re}\left(\zeta_{\alpha}\right)$ and $\operatorname{Im}\left(\zeta_{\alpha}\right) / \operatorname{Re}\left(\zeta_{\alpha}\right)$ are presented in Figures 3 to 6 . They were obtained using the approximations, Eqs. (39) and subsequent, of sec. 3.1 for the fast wave and Eqs. (43) 
and subsequent, of sec. 3.2 for the slow wave. Dashed, dotted and dotdashed lines illustrate the account of only the first order in concentration $\delta_{1}^{1}$, of going up to the second order in concentration without mode conversions $\delta_{2}^{1, c}$, and finally of going up to the second order in concentration with inclusion of mode conversions $\delta_{2}^{1,0}+\delta_{2}^{1, c}$. As it can be seen, mode conversions take place in a meaningful way whatever the frequency. The inclusion of metallic spheres in the QF20 causes significant modifications in the fast wave : (i) its phase velocity increases of about $160 \mathrm{~m} / \mathrm{s}$ for frequencies lower than $1 \mathrm{kHz}$, (ii) the peak of attenuation decreases in magnitude from 0.0086 to about 0.0079.

The effect of the metallic spheres is less substantial for the slow wave. Its phase velocity and attenaution are weakly sensitive to the presence of spheres. The various orders of concentration show nonetheless in the $1-50 \mathrm{kHz}$ frequency range a decrease in velocity, Fig. 5. Concerning the attenuation of the slow wave, the changes are not perceptible in Fig. 6. Note that the curves of the velocities and attenuations of the effective wavenumbers are drawn according to the approximations we have provided. Although these approximations are in principle limited in precision, they do not differ significantly in their appearance from the fast and slow wave velocities and attenuations in the QF20 without spheres.

The result of replacing aluminum by epoxy which is a softer material is presented in Fig. 7 for the fast wave. The comparison with Fig. 3 shows clearly the influence of the material constitutive of the spheres. The presence of epoxy spheres lowers the attenuation and increases the velocity. For the slow wave (not shown here), the change of properties for the spheres does not affect neither the velocity nor the attenuation (virtually nothing is modified 
compared to the case with the aluminum spheres).

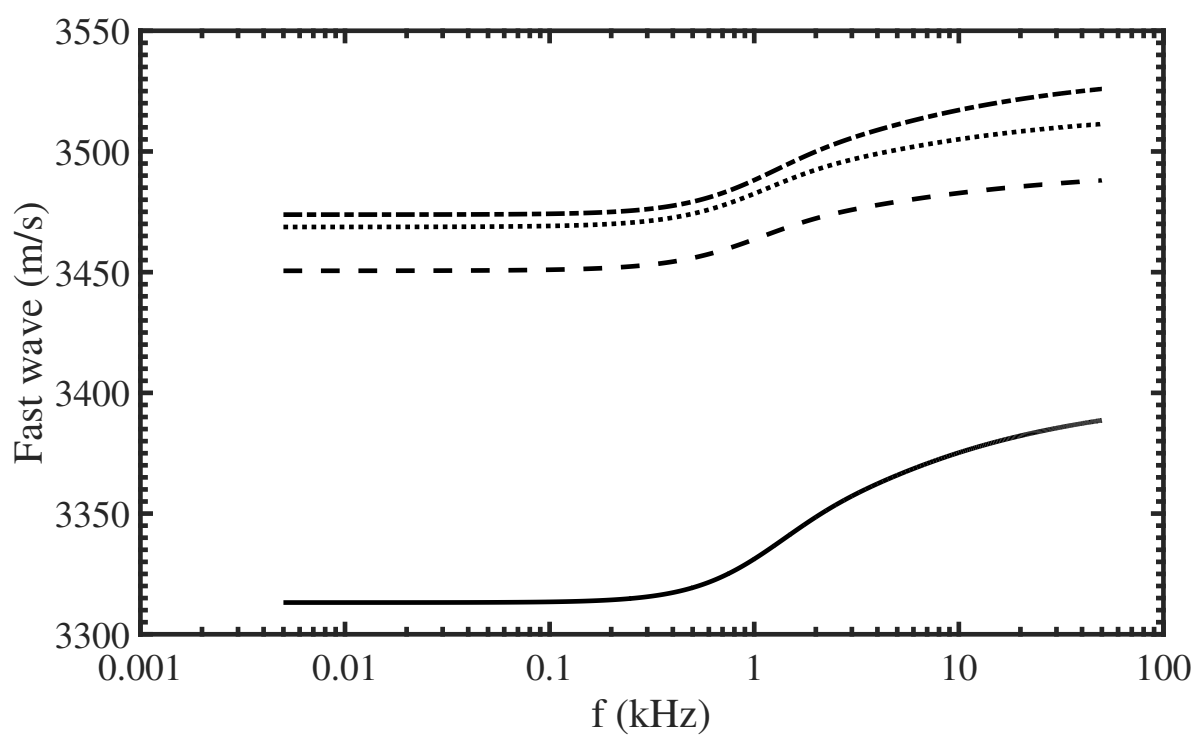

Figure 3: Fast wave phase velocities in a fluid-saturated poroelastic medium (QF20) containing randomly distributed aluminum spheres of radius $R=4 \times 10^{-3} \mathrm{~m}$ with a particle volume fraction $\mathfrak{C}=20 \%$. Matrix free of spheres (solid), considering only the first order in concentration (dashed), going up to the second order of concentration with only $\delta_{2}^{1,0}$ (dotted) and finally going up to the second order of concentration with $\delta_{2}^{1,0}+\delta_{2}^{1, c}$ (dotdashed). 


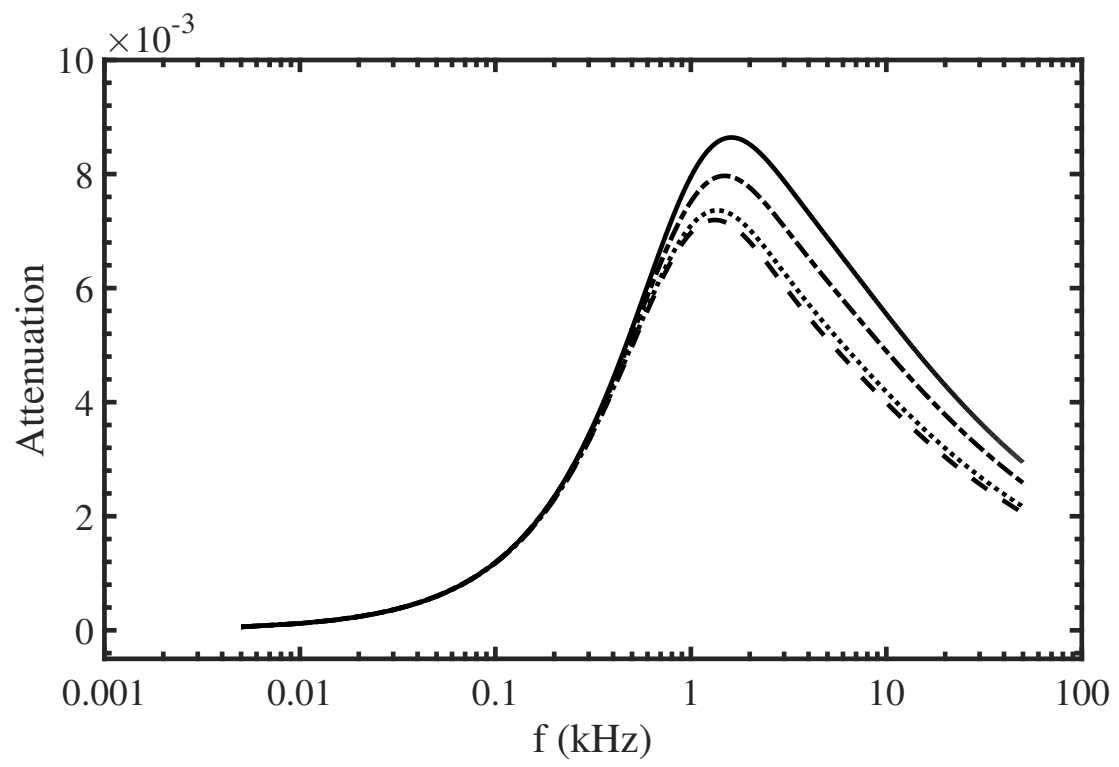

Figure 4: Fast wave attenuations in a fluid-saturated poroelastic medium (QF20) containing randomly distributed aluminum spheres of radius $R=4 \times 10^{-3} \mathrm{~m}$ with a particle volume fraction $\mathfrak{C}=20 \%$. Solid, dashed, dotted and dotdashed lines (see Fig. 5 for correspondences). 


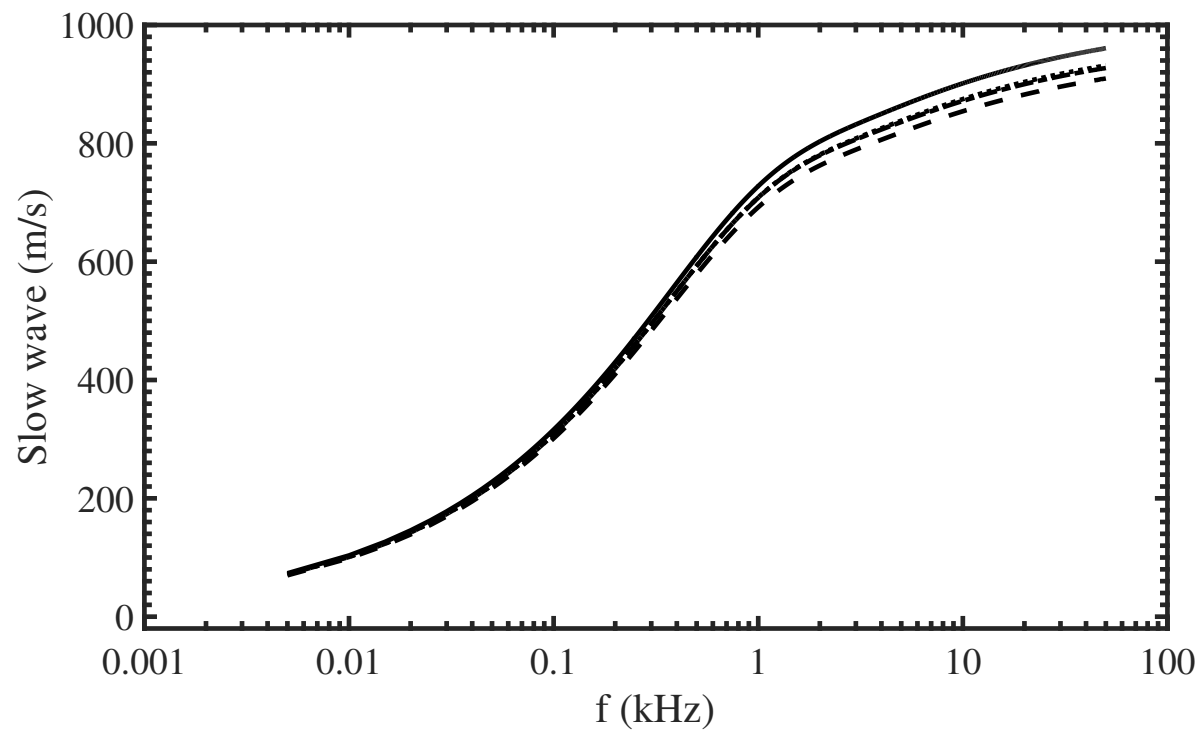

Figure 5: Slow wave phase velocities in a fluid-saturated poroelastic medium (QF20) containing randomly distributed aluminum spheres of radius $R=4 \times 10^{-3} \mathrm{~m}$ with a particle volume fraction $\mathfrak{C}=20 \%$. Matrix free of spheres (solid), considering only the first order in concentration (dashed), going up to the second order of concentration with only $\delta_{2}^{1,0}$ (dotted) and finally going up to the second order of concentration with $\delta_{2}^{1,0}+\delta_{2}^{1, c}$ (dotdashed). 


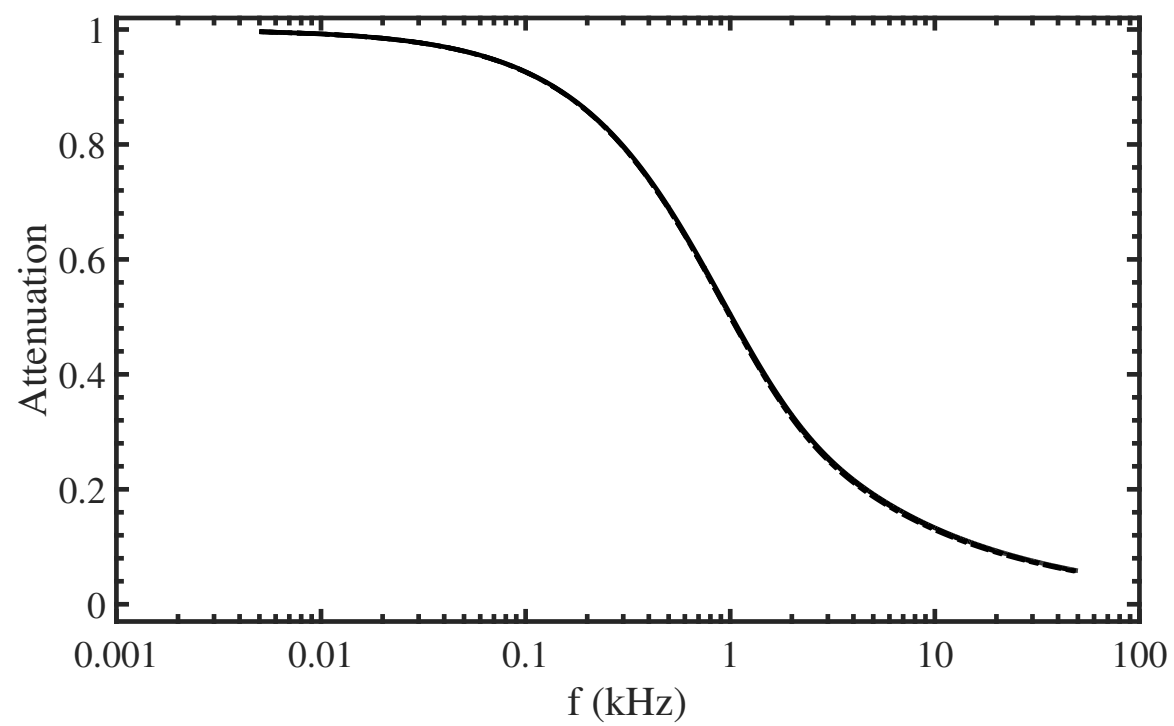

Figure 6: Slow wave attenuations in a fluid-saturated poroelastic medium (QF20) containing randomly distributed aluminum spheres of radius $R=4 \times 10^{-3} \mathrm{~m}$ with a particle volume fraction $\mathfrak{C}=20 \%$. Solid, dashed, dotted and dotdashed lines are confounded (see Fig. 5 for correspondences) . 


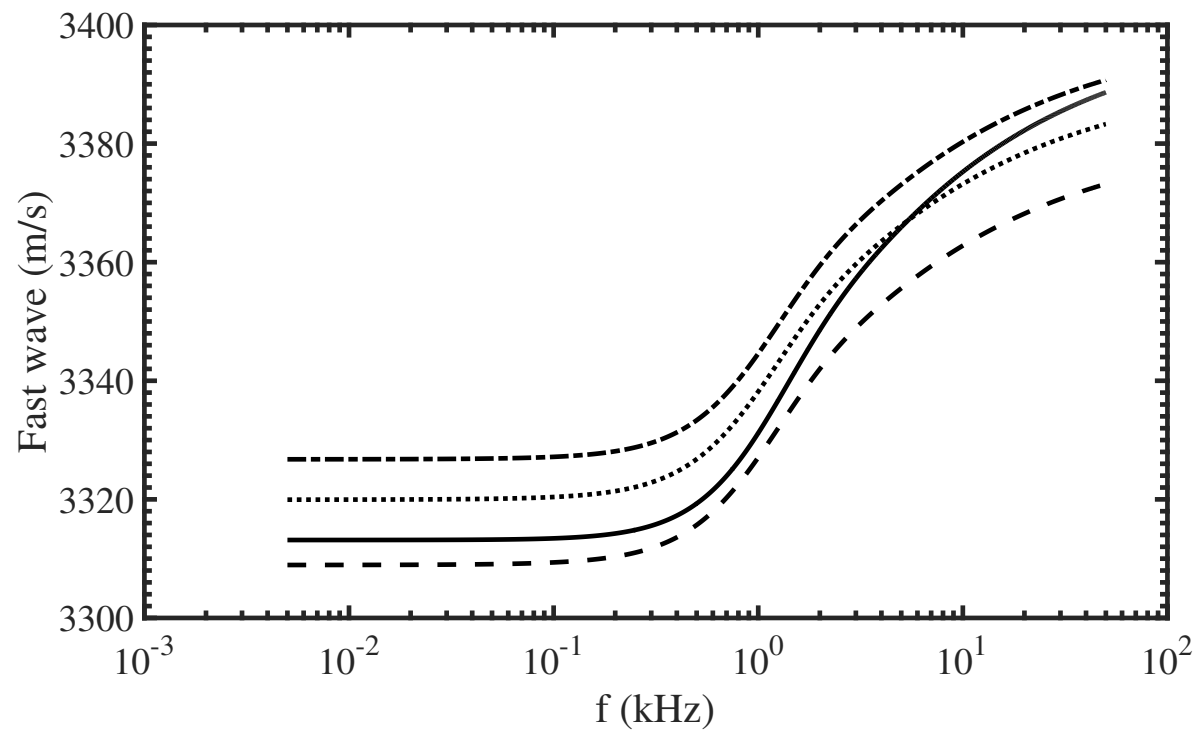

Figure 7: Fast wave phase velocities in a fluid-saturated poroelastic medium (QF20) containing randomly distributed epoxy spheres of radius $R=4 \times 10^{-3} \mathrm{~m}$ with a particle volume fraction $\mathfrak{C}=20 \%$. Matrix free of spheres (solid), considering only the first order in concentration (dashed), going up to the second order of concentration with only $\delta_{2}^{1,0}$ (dotted) and finally going up to the second order of concentration with $\delta_{2}^{1,0}+\delta_{2}^{1, c}$ (dotdashed). 


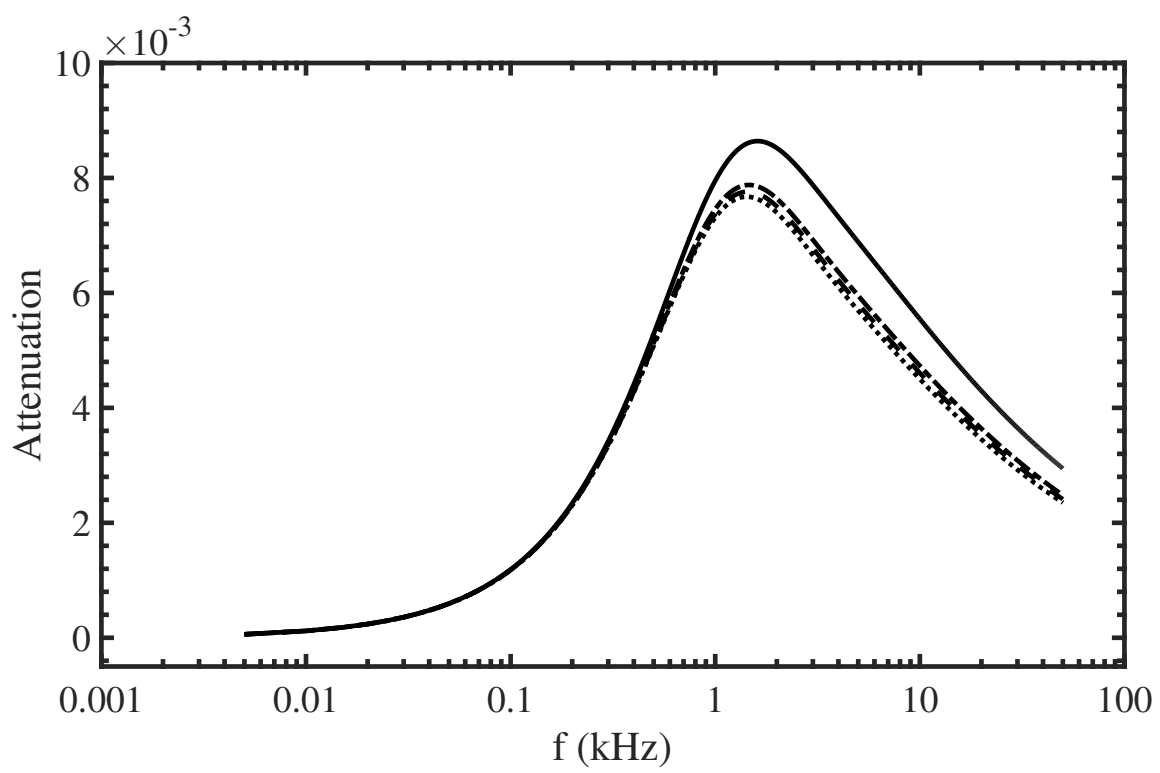

Figure 8: Fast wave phase attenuations in a fluid-saturated poroelastic medium (QF20) containing randomly distributed epoxy spheres of radius $R=4 \times 10^{-3} \mathrm{~m}$ with a particle volume fraction $\mathfrak{C}=20 \%$. Solid, dashed, dotted and dotdashed lines (see Fig. 7 for correspondences).

\section{Explicit low frequency expressions for the effective wavenumbers}

At the end of sec. 3, formulas have been found for the various constitutive terms of the fast and slow effective wavenumbers. These formulas were expressed in terms of dimensionless quantities $x_{\alpha}$ and $\gamma_{\alpha}(\alpha=1,2, t)$ depending on the angular frequency $\omega$. The status of these formulas was the provision of approximate formulas for the effective wavenumbers valid in the relatively low frequency domain where the dispersive effects take place. Now, the objective is to go a step further to formulas of effective wavenumbers close to the static limit (dispersive effects are then left aside) in order to extract effective quantities describing the composite medium. In this second step of 
approximations, the low frequency expansions of the wavenumbers $k_{\alpha}$ and of the coefficients $\gamma_{\alpha}$ are introduced in the formulas. Let $\omega_{c}=\frac{\eta}{\rho_{f} \kappa}(=67.85$ $\mathrm{kHz}$ with data of Table 1) represent a characteristic angular frequency. It is recalled at first that as $\omega$ becomes small, that is $\omega \ll \omega_{c}$, the wavenumbers take the forms $k_{1}^{2}=\frac{\omega^{2} \rho}{H}+O\left(\omega^{3}\right), k_{2}^{2}=\omega \epsilon-\omega^{2}\left(\frac{m H+\rho M-2 \rho_{f} C}{C^{2}-H M}+\frac{\rho}{H}\right)+O\left(\omega^{3}\right)$, $k_{t}^{2}=\frac{\omega^{2} \rho}{\mu}+O\left(\omega^{3}\right)$, where

$$
\epsilon=\frac{i \omega_{c} \rho_{f} H}{H M-C^{2}} .
$$

It follows that $\gamma_{1}=O(\omega), \gamma_{2}=-\frac{H}{C}+\left(\frac{\rho}{C \epsilon}-\frac{H \rho_{f}}{C^{2} \epsilon}\right) \omega+O\left(\omega^{2}\right), \gamma_{t}=O(\omega)$ and that $\mathcal{H}_{1}=H-2 \mu+O(\omega), \mathcal{H}_{2}=-2 \mu+\left(\frac{\rho}{\epsilon}-\frac{H \rho_{f}}{C \epsilon}\right) \omega+O\left(\omega^{2}\right)$. The constants $H, M$ and $C$ are detailed in the Appendix. In the light from above, we get

$$
\begin{gathered}
b=\omega\left(1-\frac{H \rho_{f}}{C \rho}\right) \frac{\rho}{\epsilon}\left(\mu-\mu_{s}\right)+O\left(\omega^{2}\right), \quad c=H\left(\mu-\mu_{s}\right)+O(\omega), \\
d=\omega\left(1-\frac{H \rho_{f}}{C \rho}\right) \frac{\rho}{\epsilon}\left(2 \mu+3 \mu_{s}\right)+O\left(\omega^{2}\right), \quad e=H\left(2 \mu+3 \mu_{s}\right)+O(\omega),
\end{gathered}
$$

and

$$
\gamma_{21}=\frac{-H}{C}+O(\omega), \quad \gamma_{t 1}=O(\omega), \quad \gamma_{2 t}=\frac{-H}{C}+O(\omega) .
$$

Therefore, as $\omega \rightarrow 0$, for the mode $n=0$

$$
\frac{b_{0}^{11}}{d_{0}} \rightarrow \frac{3 H}{3 \lambda_{s}+4 \mu+2 \mu_{s}},
$$

and

$$
\left(\frac{b_{0}^{12}}{d_{0}}, \frac{b_{0}^{21}}{d_{0}}, \frac{b_{0}^{22}}{d_{0}}\right) \rightarrow(0,0,0),
$$

while for the mode $n=1$

$$
\begin{gathered}
\left(\frac{b_{1}^{11}}{d_{1}}, \frac{b_{1}^{12}}{d_{1}}, \frac{b_{1}^{1 t}}{d_{1}}\right) \rightarrow\left(\frac{\rho-\rho_{s}}{3 \rho}, 0, \frac{\rho-\rho_{s}}{3 \rho}\right) \\
\left(\frac{b_{1}^{21}}{d_{1}}, \frac{b_{1}^{22}}{d_{1}}, \frac{b_{1}^{2 t}}{d_{1}}\right) \rightarrow\left(\frac{\rho-\rho_{s}}{3 \rho}+\frac{3}{2}\left(1-\frac{H \rho_{f}}{C \rho}\right),-\frac{1}{2},-\frac{\rho+2 \rho_{s}}{6 \rho}+\frac{1}{2}\left(1-\frac{H \rho_{f}}{C \rho}\right)\right),
\end{gathered}
$$




$$
\left(\frac{b_{1}^{t 1}}{d_{1}}, \frac{b_{1}^{t 2}}{d_{1}}, \frac{b_{1}^{t t}}{d_{1}}\right) \rightarrow 2\left(\frac{\rho-\rho_{s}}{3 \rho}, 0, \frac{\rho-\rho_{s}}{3 \rho}\right),
$$

and

$$
L^{1 t} \rightarrow \frac{2(\mu / H)^{\frac{3}{2}}}{(\mu / H)^{2}-1} .
$$

For the various $\delta$ terms - Eq. (39) and subsequent - involved in the fast effective wavenumber, we obtain to the first approximation

$$
\begin{gathered}
n_{0} \delta_{1}^{1}=\omega^{2} \frac{\rho}{H} \mathfrak{C}\left[\frac{3 H}{3 \lambda_{s}+2 \mu_{s}+4 \mu}-1-\frac{\rho-\rho_{s}}{\rho}\right] \\
n_{0}^{2} \delta_{2}^{1,0}=\omega^{2} \frac{\rho}{H} \mathfrak{C}^{2}\left[\left(1-\frac{3 H}{3 \lambda_{s}+2 \mu_{s}+4 \mu}\right) \frac{\rho-\rho_{s}}{\rho}+\left(\frac{\rho-\rho_{s}}{3 \rho}\right)^{2}\right],
\end{gathered}
$$

and

$$
n_{0}^{2} \delta_{2}^{1, c}=\frac{1}{2} \omega^{2} \frac{\rho}{H} \mathfrak{C}^{2} L^{1 t}\left(1+2 \frac{\mu}{H}\right)\left(\frac{H}{\mu}\right)^{\frac{3}{2}}\left(\frac{\rho-\rho_{s}}{3 \rho}\right)^{2} .
$$

For the $\delta$ terms - Eqs. (43) and subsequent - useful to built the slow effective wavenumber, we obtain to the first approximation

$$
\begin{aligned}
n_{0} \delta_{1}^{2} & =\frac{1}{2} \omega \epsilon \mathfrak{C}, \\
n_{0}^{2} \delta_{2}^{2,0} & =\frac{-5}{4} \omega \epsilon \mathfrak{C}^{2},
\end{aligned}
$$

and

$$
n_{0}^{2} \delta_{2}^{2, c}=O\left(\omega^{2}\right)
$$

In the fast wave, the $\delta$ s depend both on the parameters of the matrix and the spheres. Moreover, $\delta_{2}^{1, c}$ is of the same order as $\delta_{2}^{1,0}$ showing that at low frequency the mode conversions $1 \leftrightarrow 2$ and $1 \leftrightarrow t$ are as important as the non-conversions $1 \leftrightarrow 1$ in the fast effective wavenumber.

In the slow wave, the first terms in the expansion of the $\delta$ s only depend on the poroelastic medium through $\epsilon$. The first non-zero term obtained for $\delta_{2}^{2, c}$ is $O\left(\omega^{2}\right)$, Eq. (61). At low frequency, because $\left|\delta_{2}^{2, c}\right| \ll\left|\delta_{2}^{2,0}\right|$, the mode conversions $2 \leftrightarrow 1$ and $2 \leftrightarrow t$ participate very few in the slow wavenumber. 


\section{Effective bulk modulus and mass density}

In this section and the next, we investigate the effective moduli, densities and diffusion coefficient of the composite material. The formulas obtained are general and allow an independent discussion of the numerical data of Table 1. For example, in sec. 6, the limits where the porosity is zero or equal to one are examined, while in sec. 7, The effects of skeletal elasticity on the slow wave are discussed. Let us expand the ratio of the fast wavenumbers as $\frac{\zeta_{1}^{2}}{k_{1}^{2}}=\frac{\zeta_{1}^{2}}{\omega^{2}} \frac{\omega^{2}}{k_{1}^{2}}$. For $\omega \ll \omega_{c}$, let us also admit that $\zeta_{1}^{2}$ must behave like $k_{1}^{2}$, that is $\zeta_{1}^{2}=\frac{\omega^{2} \rho_{1, e}}{H_{1, e}}$ where $\rho_{1, e}$ and $H_{1, e}$ denote the effective mass density and

bulk modulus, respectively. Thence, $\frac{\zeta_{1}^{2}}{k_{1}^{2}}=\frac{\rho_{1, e}}{\rho} \frac{H}{H_{1, e}}$. Identifying next with the factor decomposition Eq. (42), we deduce the following expressions

$$
\rho_{1, e}=\rho\left(1-3 \mathfrak{C} \frac{b_{1}^{11}}{d_{1}}\right)=(1-\mathfrak{C})\left[(1-\phi) \bar{\rho}_{s}+\phi \rho_{f}\right]+\mathfrak{C} \rho_{s},
$$

and

$$
\frac{H}{H_{1, e}}=1+\mathfrak{C}\left(\frac{b_{0}^{11}}{d_{0}}-1\right)=1+\mathfrak{C}\left(\frac{3 H}{3 \lambda_{s}+2 \mu_{s}+4 \mu}-1\right) .
$$

An alternative form of this last relation is

$$
\frac{1}{H_{1, e}}=\frac{1-\mathfrak{C}}{H}+\frac{\mathfrak{C}}{\lambda_{s}+\frac{2}{3} \mu_{s}+\frac{4}{3} \mu} .
$$

For a low porosity matrix $(\phi \rightarrow 0)$, we have $\rho \rightarrow \bar{\rho}_{s}, H \rightarrow \bar{K}_{s}+\frac{4}{3} \bar{\mu}_{s}\left(K_{b} \rightarrow \bar{K}_{s}\right.$, $\left.\mu \rightarrow \bar{\mu}_{s}, \sigma \rightarrow 0\right)$. It follows that

$$
\begin{gathered}
\frac{\rho_{1, e}}{\rho} \rightarrow \frac{\rho_{e l, e}}{\bar{\rho}_{s}}=1-\mathfrak{C} \frac{\bar{\rho}_{s}-\rho_{s}}{\bar{\rho}_{s}}, \\
\frac{1}{H_{1, e}} \rightarrow \frac{1}{H_{e l, e}}=\frac{1-\mathfrak{C}}{\bar{K}_{s}+\frac{4}{3} \bar{\mu}_{s}}+\frac{\mathfrak{C}}{K_{s}+\frac{4}{3} \overline{\mu_{s}}},
\end{gathered}
$$

where $\rho_{e l, e}$ and $H_{e l, e} \equiv K_{e l, e}+\frac{4}{3} \mu_{e l, e}$ represent effective quantities for the distribution of elastic spheres buried in an elastic matrix (barred quantities 
are for the matrix). For $\rho_{s}<\bar{\rho}_{s}$, we get $\rho_{1, e} / \rho_{s}<1$, i.e. an effective medium less dense than the elastic host medium. The shear modulus $\mu_{s}$ of the spheres is absent in Eq. (66). Thus, two phases (matrix and sphere) equal by their bulk moduli but different by their shear moduli lead to the same effective modulus. If in the left hand side of Eq. (66), $\mu_{e l, e}$ is replaced by $\bar{\mu}_{s}$, the resulting formula looks like the Kuster-Töksoz estimate for two constituants with particle volume concentrations $\mathfrak{C}$ and $1-\mathfrak{C}$, Eq.(19) of Ref. [42].

For a high porosity matrix $(\phi \rightarrow 1)$, we have $\rho \rightarrow \rho_{f}, H \rightarrow K_{f}\left(K_{b} \rightarrow 0\right.$, $\mu \rightarrow 0, \sigma \rightarrow 1)$ from which follow the limits

$$
\begin{gathered}
\frac{\rho_{1, e}}{\rho} \rightarrow \frac{\rho_{\text {liq,e }}}{\rho_{f}}=1-\mathfrak{C} \frac{\rho_{f}-\rho_{s}}{\rho_{f}}, \\
\frac{1}{H_{1, e}} \rightarrow \frac{1}{H_{\text {liq,e }}}=\frac{1-\mathfrak{C}}{K_{f}}+\frac{\mathfrak{C}}{K_{s}},
\end{gathered}
$$

where $\rho_{l i q, e}$ and $H_{l i q, e} \equiv K_{l i q, e}$ are effective mass density and bulk modulus for the distribution of elastic spheres in a liquid. Equation (68) is typical of the Reuss average [25], found by assuming that the stress at every position of the effective medium is equal to the macroscopic stress of the sample.

\section{Effective diffusion coefficient}

In a Biot poroelastic medium, the conditions that favor the propagation of a slow high amplitude wave are as follows : continuity of the two phases (fluid and solid), high acoustic frequency, high fluid permeability and low viscosity [43], [44]. At low frequency $\left(\omega<<\omega_{c}\right)$, the slow wave is of the diffusive type, i.e. governed by a diffusion equation with a hydraulic diffusivity coefficient. With these facts in mind, we now deal with the slow effective wavenumber. 
By using the results of sec. 5 , it can be shown that :

$$
\zeta_{2}^{2}=\frac{i \omega}{\chi}\left(1+\frac{1}{2} \mathfrak{C}-\frac{5}{4} \mathfrak{C}^{2}\right)
$$

where

$$
\chi=i \epsilon^{-1}=\frac{H M-C^{2}}{\omega_{c} \rho_{f} H} .
$$

This result suggests a diffusion process in the time domain governed by the partial differential equation

$$
\mathcal{D}_{e} \vec{\nabla}^{2} \phi_{2}=\frac{\partial \phi_{2}}{\partial \tau}
$$

where $\phi_{2}$ is a displacement potential for the slow wave (with time dependence $\left.e^{-i \omega \tau}\right)$ and

$$
\mathcal{D}_{e}=\frac{i \omega}{\zeta_{2}^{2}}=\frac{\chi}{1+\frac{1}{2} \mathfrak{C}-\frac{5}{4} \mathfrak{C}^{2}}
$$

the effective diffusion coefficient. The mechanical stability requierement $H M-C^{2} \geq 0$ imposes $\mathcal{D}_{e}>0$. On the other hand, since $\mathfrak{C}<\frac{2}{5}$ (in the frame of the dilute case imposed all along the paper for using the LCN formula), the effective diffusion coefficient is such that $\mathcal{D}_{e}<\left.\mathcal{D}_{e}\right|_{\mathfrak{C}=0}=\chi$. Clearly, the presence of elastic spheres in the poroelastic matrix lowers the diffusion coefficient. The expression of $\left.\mathcal{D}_{e}\right|_{\mathfrak{C}=0}$ was given by Berryman, see Eq. (73) of Ref. [3].

Note that "the soft sediment case" where $H M-C^{2} \approx 0$ yields $\mathcal{D}_{e} \approx 0$, a value also obtained for high porosity frames where $H \approx M \approx C$. If the poroelastic matrix is made of regular spherical grains, the permeability is generally approximated by the Kozeny-Carman formula $\kappa=\frac{\phi a_{p}^{2}}{20}$ where $a_{p}=\frac{\phi d_{g}}{3(1-\phi)}$ is the pore size parameter corresponding to spherical grains of diameter $d_{g}$. 
For an infinitely rigid frame $\left(H \rightarrow \infty, M \rightarrow \frac{K_{f}}{\phi}\right)$

$$
\mathcal{D}_{e} \rightarrow \mathcal{D}_{e}^{r i g}=\frac{K_{f} a_{p}^{2}}{20 \eta} \frac{1}{1+\frac{1}{2} \mathfrak{C}^{\mathfrak{c}}-\frac{5}{4} \mathfrak{C}^{2}},
$$

with $\mathcal{D}_{e}^{\text {rig }}$ tending to zero as $a_{p}$ or the porosity tends to zero. From Eq. (69) and by retaining only the positive values for the real part, we find that

$$
\zeta_{2}=(1+i) \sqrt{\frac{\omega}{2 \chi}\left(1+\frac{1}{2} \mathfrak{C}-\frac{5}{4} \mathfrak{C}^{2}\right)} .
$$

This result shows that the slow wave is quickly damped inside the effective porous medium. The damping depends on the concentration. The distance at which the amplitude is reduced by $e$ times is

$$
\Delta_{e}=\sqrt{\frac{2 \chi}{\omega\left(1+\frac{1}{2} \mathfrak{C}-\frac{5}{4} \mathfrak{C}^{2}\right)}},
$$

an effective depth of penetration. With data of Table 1 and $f=10 \mathrm{kHz}$, $\Delta_{e}=4.5 \times 10^{-2} \mathrm{~m}$ for $\mathfrak{C}=20 \%$. This value can be compared with other

characteristic lengths, namely the viscous skin depth $d_{s}=\sqrt{\frac{2 \eta}{\omega \rho}}=4.2 \times$ $10^{-6} \mathrm{~m}$, the mean pore radius $3.26 \times 10^{-5} \mathrm{~m}$ and the radius of the spheres $4 \times 10^{-3} \mathrm{~m}$. Since $\mathfrak{C}=n_{0}\left(4 \pi R^{3} / 3\right)$, the larger the size of the scatterers, the smaller $\Delta_{e}$ for a fixed number of spheres per unit volume. To conclude this section, we can note the absence of contribution from the $\delta_{2}^{2, c}$ term for wave conversions. Such a result is due to the fact that we considered the lower terms of the expansions in powers of $\omega$. Further calculations should make it possible to introduce the effects of conversions.

\section{Conclusion}

Fast and slow waves behave very differently from each other in a poroelastic medium obeying Biot's theory. The fast wave is propagative at all 
frequencies, while the slow wave is diffusive at very low frequencies, only becoming propagative when the frequency is sufficiently high. In the presence of spherical scatterers distributed randomly in the poroelastic medium, the frequency behaviors of the fast and slow waves remain but are nevertheless influenced by the concentration of scatterers. From Luppe, Conoir and Norris (LCN) effective wavenumber formula extended to the poroelastic case, we derived expressions for the fast and slow effective wave numbers at low frequency. On the one hand, the analysis of the effective fast wave allowed us to highlight the importance of the mode conversions and to extract the effective bulk modulus and mass density of the poroelastic composite medium. On the other hand, the analysis of the slow wave allowed us to extract an effective diffusion coefficient depending on the particle volume fraction (up to the second order) and to estimate a penetration depth. At low frequency, the physical properties of the spheres have very little influence on the effective properties of the slow wave.

Potential working applications are expected to be in particulate composites and metamaterials. The use of a poroelastic matrix instead of an elastic matrix has the effect of introducing low frequency absorption. In this, the present study also offers an alternative to the use of viscoelastic matrices. Viscoelastic materials have fixed properties whereas a poroelastic medium can undergo transformations acting on the acoustic characteristics: modification of the porosity (therefore of the permeability) via invasion or erosion processes, modification of the scatterers by a chemical process, modification of the saturating fluid. In fact, poroelastic matrices (the skeleton of which can be made with a viscoelastic material) could allow the advent of new types 
of metamaterials with modifiable performance at will. 


\section{Appendix : Derivation of the scattering coefficients}

Let $(r, \theta, \varphi)$ be spherical coordinates with orthonormal basis vectors $\left(\vec{e}_{r}\right.$, $\left.\vec{e}_{\theta}, \vec{e}_{\varphi}\right)$. Consider an elastic isotropic sphere of radius $R$ placed at the origin of the axes. In this paper, $\rho_{s}, \lambda_{s}$ and $\mu_{s}$ denote the mass density and the Lamé constants of the constitutive material. Let $u_{s r}, u_{s \theta}$ be the radial and tangential displacements. Let also $\sigma_{s r r}, \sigma_{s r \theta}$ be radial and tangential components of the stress tensor in the sphere. The wavenumber of the longitudinal wave is $k_{L}=\omega / c_{L}$, that of the transverse wave $k_{T}=\omega / c_{T}$, where

$c_{L}=\sqrt{\left(\lambda_{s}+2 \mu_{s}\right) / \rho_{s}}$ and $c_{T}=\sqrt{\mu_{s} / \rho_{s}}$ denote velocities and $\omega$ the angular frequency.

The sphere is embedded in a poroelastic isotropic medium obeying Biot's theory (a full account of the theory can be found in Refs. [1], [45] and [3]). Wavelengths are assumed very large compared to the pore size, so that average values of the local displacements in the solid and the fluid are accounted for. Let $u_{r}$ and $u_{\theta}$ be the displacement components of the frame, and $w_{r}$ and $w_{\theta}$ be those of the relative motion of the fluid with respect to the solid. The components of the stress tensor are denoted by $\sigma_{r r}$ and $\sigma_{r \theta}$. The poroelastic medium sustains two longitudinal waves with respective wavenumber $k_{1}$ (fast wave) and $k_{2}$ (slow wave), and a transverse wave with wavenumber $k_{t}$.

The application of the boundary conditions $u_{s r}=u_{r}, u_{s \theta}=u_{\theta}, w_{r}=0$, $\sigma_{s r r}=\sigma_{r r}$ and $\sigma_{s r \theta}=\sigma_{r \theta}$ at $r=R$ yields a linear system

$$
M_{n} \vec{X}_{n}^{\alpha}=\vec{S}_{n}^{\alpha}
$$

of 5 equations with 5 unknowns where the superscript $\alpha$ indicates the type 
of the incident wave $(\alpha=1,2$ or $t)$. The elements of the $5 \times 5$ matrix $M_{n}$ are given by

$$
\begin{aligned}
& m_{n, 11}=x_{1} h_{n}^{\prime}\left(x_{1}\right) \\
& m_{n, 12}=x_{2} h_{n}^{\prime}\left(x_{2}\right) \\
& m_{n, 13}=n(n+1) h_{n}\left(x_{t}\right) \\
& m_{n, 14}=-x_{L} j_{n}^{\prime}\left(x_{L}\right) \\
& m_{n, 15}=-n(n+1) j_{n}\left(x_{T}\right) \\
& m_{n, 21}=h_{n}\left(x_{1}\right) \\
& m_{n, 22}=h_{n}\left(x_{2}\right) \\
& m_{n, 23}=h_{n}\left(x_{t}\right)+x_{t} h_{n}^{\prime}\left(x_{t}\right) \\
& m_{n, 24}=-j_{n}\left(x_{L}\right) \\
& m_{n, 25}=-x_{T} j_{n}^{\prime}\left(x_{T}\right)-j_{n}\left(x_{T}\right) \\
& m_{n, 31}=\gamma_{1} x_{1} h_{n}^{\prime}\left(x_{1}\right) \\
& m_{n, 32}=\gamma_{2} x_{2} h_{n}^{\prime}\left(x_{2}\right) \\
& m_{n, 33}=\gamma_{t} n(n+1) h_{n}\left(x_{t}\right) \\
& m_{n, 34}=0 \\
& m_{n, 35}=0 \\
& m_{n, 41}=\left[-\mathcal{H}_{1} x_{1}^{2}+2 \mu\left(n(n+1)-x_{1}^{2}\right)\right] h_{n}\left(x_{1}\right)-4 \mu x_{1} h_{n}^{\prime}\left(x_{1}\right) \\
& m_{n, 42}=\left[-\mathcal{H}_{2} x_{2}^{2}+2 \mu\left(n(n+1)-x_{2}^{2}\right)\right] h_{n}\left(x_{2}\right)-4 \mu x_{2} h_{n}^{\prime}\left(x_{2}\right) \\
& m_{n, 43}=2 \mu n(n+1)\left[-h_{n}\left(x_{t}\right)+x_{t} h_{n}^{\prime}\left(x_{t}\right)\right] \\
& m_{n, 44}=-\left[-\lambda_{s} x_{L}^{2}+2 \mu_{s}\left(n(n+1)-x_{L}^{2}\right)\right] j_{n}\left(x_{L}\right)+4 \mu_{s} x_{L} j_{n}^{\prime}\left(x_{L}\right) \\
& =-2 \mu_{s} n(n+1)\left[-j_{n}\left(x_{T}\right)+x_{T} j_{n}^{\prime}\left(x_{T}\right)\right] \\
& =
\end{aligned}
$$




$$
\begin{aligned}
& m_{n, 51}=2 \mu\left[-h_{n}\left(x_{1}\right)+x_{1} h_{n}^{\prime}\left(x_{1}\right)\right], \\
& m_{n, 52}=2 \mu\left[-h_{n}\left(x_{2}\right)+x_{2} h_{n}^{\prime}\left(x_{2}\right)\right], \\
& m_{n, 53}=2 \mu\left[-x_{t} h_{n}^{\prime}\left(x_{t}\right)+\left(n(n+1)-1-\frac{x_{t}^{2}}{2}\right) h_{n}\left(x_{t}\right)\right], \\
& m_{n, 54}=-2 \mu_{s}\left[-j_{n}\left(x_{L}\right)+x_{L} j_{n}^{\prime}\left(x_{L}\right)\right], \\
& m_{n, 55}=-2 \mu_{s}\left[-x_{T} j_{n}^{\prime}\left(x_{T}\right)+\left(n(n+1)-1-\frac{x_{T}^{2}}{2}\right) j_{n}\left(x_{T}\right)\right],
\end{aligned}
$$

If $\alpha=1$ (incidence of the fast longitudinal wave ) or $\alpha=2$ (incidence of the slow longitudinal wave)

$$
\vec{S}_{n}^{\alpha}=\left(\begin{array}{c}
-x_{\alpha} j_{n}^{\prime}\left(x_{\alpha}\right) \\
-j_{n}\left(x_{\alpha}\right) \\
-\gamma_{\alpha} x_{\alpha} j_{n}^{\prime}\left(x_{\alpha}\right) \\
-\left[-\mathcal{H}_{\alpha} x_{\alpha}^{2}+2 \mu\left(n(n+1)-x_{\alpha}^{2}\right)\right] j_{n}\left(x_{\alpha}\right)+4 \mu x_{\alpha} j_{n}^{\prime}\left(x_{\alpha}\right) \\
-2 \mu\left[-j_{n}\left(x_{\alpha}\right)+x_{\alpha} j_{n}^{\prime}\left(x_{\alpha}\right)\right] .
\end{array}\right)
$$

and if $\alpha=t$ (transverse wave incidence)

$$
\vec{S}_{n}^{t}=\left(\begin{array}{c}
-n(n+1) j_{n}\left(x_{t}\right) \\
-j_{n}\left(x_{t}\right)-x_{t} j_{n}^{\prime}\left(x_{t}\right) \\
-\gamma_{t} n(n+1) j_{n}\left(x_{t}\right) \\
-2 \mu n(n+1)\left[-j_{n}\left(x_{t}\right)+x_{t} j_{n}^{\prime}\left(x_{t}\right)\right] \\
-2 \mu\left[-x_{t} j_{n}^{\prime}\left(x_{t}\right)+\left(n(n+1)-1-\frac{x_{t}^{2}}{2}\right) j_{n}\left(x_{t}\right)\right]
\end{array}\right) .
$$

The vector $\vec{X}_{n}^{\alpha}$ contains the unknown scattering coefficients $t_{n}^{\alpha \beta}$ by one sphere in relation with the type of the incident $(\alpha)$ and scattered $(\beta)$ waves and can be written as

$$
\vec{X}_{n}^{\alpha}=\left(\begin{array}{lllll}
t_{n}^{\alpha 1} & t_{n}^{\alpha 2} & t_{n}^{\alpha t} & B_{n}^{\alpha L} & B_{n}^{\alpha T}
\end{array}\right)^{T}
$$


Above, $x_{j}=k_{j} R(j=1,2, t, L, T)$ are dimensionless wavenumbers and primes over the spherical Bessel functions $j_{n}$ and Hankel functions $h_{n}\left(\equiv h_{n}^{(1)}\right)$ denote derivatives with respect to the arguments. The scattering coefficients $t_{n}^{\alpha \beta}(n \in \mathbb{R}$ and $\alpha, \beta=1,2, t)$ are found by solving the linear system Eq. (A.1).

The definition of the coefficients $\gamma_{j}$ and $\gamma_{t}$ are

$$
\begin{gathered}
\gamma_{j}=\frac{H k_{j}^{2}-\rho \omega^{2}}{\rho_{f} \omega^{2}-C k_{j}^{2}},(j=1,2) \\
\gamma_{t}=\frac{\mu k_{j}^{2}-\rho \omega^{2}}{\rho_{f} \omega^{2}},
\end{gathered}
$$

where $\rho_{f}$ and $\rho=(1-\phi) \overline{\rho_{s}}+\phi \rho_{f}$ are the mass densities for the saturating fluid and the poroelastic medium, respectively. These coefficients originate from the proportionality relations between the amplitudes of the potentials that are necessary for the description of the displacement fields in the fluid and solid phases, see for example Ref. [45]. Note also that

$$
\mathcal{H}_{j}=H-2 \mu+\gamma_{j} C
$$

for $j=1,2$. The moduli $H, C, \mu$ (and $M$ ) of the fluid-saturated poroelastic medium fulfill the mechanical stability requirements $H \geq 0, M \geq 0$, $\mu \geq 0$ and $H M-C^{2} \geq 0$. Brown and Korringa [46] have shown that $H=K_{b}+\sigma C+\frac{4}{3} \mu, C=\sigma\left[\sigma / \bar{K}_{s}+\phi\left(1 / K_{f}-1 / \bar{K}_{s}\right)\right]^{-1}$ and $M=C / \sigma$ where $\sigma=1-K_{b} / \bar{K}_{s}$. 


\section{References}

[1] M. A. Biot, Theory of propagation of elastic waves in a fluid-saturated porous solid. I. Low frequency range, J. Acoust. Soc. Am. 28 (1956) 168 $-178$

[2] M. A. Biot, Theory of propagation of elastic waves in a fluid-saturated porous solid. II. Higher frequency range, J. Acoust. Soc. Am. 28 (1956) $179-191$

[3] J. G. Berryman, Elastic wave propagation in fluid saturated porous media, J. Acoust. Soc. Am. 69 (2) (1981) 416-424

[4] P. C. Waterman, Matrix theory of elastic wave scattering, J. Acoust. Soc. Am. 60 (1976) 567-580

[5] S. G. Kargl and R. Lim, A transition-matrix formulation of scattering in homogeneous, saturated porous media, J. Acoust. Soc. Am. 94 (3) (1993) $1527-1550$

[6] C. Zimmerman and Stern, Scattering of plane compressional waves by spherical inclusions in a poroelastix medium, J. Acoust. Soc. Am. 94 (1) (1993) 527-536

[7] J. G. Berryman, Scattering by a spherical inhomogeneity in a fluidsaturated porous medium, J. Math. Phys., 26 (6) (1985), pp. 1408-1419.

[8] L. L. Foldy, The multiple scattering of waves. I. General theory of isotropic scattering by randomly distributed scatterers, Phys. Rev. 67 (1945), 107-119 
[9] M. Lax, Multiple scattering of waves. II. The effective field in dense systems, Phys. Rev. 85 (4) (1952) 621-629

[10] P. C. Waterman and R. Truell, Multiple scattering of waves, J. Math. Phys. 2 (4) (1961) 512- 537

[11] J. G. Fikioris and P. C. Waterman, Multiple scattering of waves II. "Hole correstions" in the scalar case, J. Math. Phys. 5 (10) (1964), 1413-1420.

[12] P. Lloyd and M. V. Berry, Wave propagation trough an assembly of spheres. IV. Relations between different multiple scattering theories, Proc. Phys. Soc. London 91 (1967) 678- 688

[13] A. Ishimaru, Wave Propagation and Scattering in Random Media (Oxford University Press, Oxford, 1978)

[14] P. Sheng, Introduction to Wave Scattering, Localization and Mesoscopic Phenomena (Academic, New York, 1995).

[15] L. Tsang, J. A. Kong and K. H. Ding, Scattering of Electromagnetic Waves, Vol. 1: Theory and Applications (Wiley, New-York, 2000).

[16] C. M. Linton and P. A. Martin, Multiple scattering by multiple spheres : A new proof of the Lloyd-Berry formula for the effective wavenumber, SIAM J. Appl. Math. 66 (5) (2006) 1649-1668

[17] C. M. Linton and P. A. Martin, Multiple scattering by random configurations of circular cylinders: Second-order corrections for the effective wavenumber, J. Acoust. Soc. Am. 117(6), (2005) pp 3413-3423 
[18] P. A. Martin, A. Maurel, and W. J. Parnell, Estimating the dynamic effective mass density of random composites. J. Acoust. Soc. Am., 128(2), pp 571-577, August 2010.

[19] W. J. Parnell and I. D. Abrahams, Multiple point scattering to determine the effective wavenumber and effective material properties of an inhomogeneous slab, Waves in Random and Complex Media, 20 (2010), pp. 678-701, https://doi.org/10.1080/17455030.2010. 510858.

[20] A. L. Gower, M. J. A. Smith, W. J. Parnell, and I. D. Abrahams Reflection from a multi-species material and its transmitted effective wavenumber. Proc. R. Soc. A, 474(2212):20170864, April 2018

[21] G. T. Kuster and M. Nafi Toksöz, Velocity and attenuation of seismic waves in two phase media: part I. Theoretical formulation, Geophysics 39 (5) (1974) 587-606

[22] A. N. Norris, Scattering of elastic waves by spherical inclusions with applications to low frequency wave propagation in composites, Int. J. Engng. Sci. 24 (8) (1986) 1271-1282

[23] V. K. Varadan, Y. Ma and V. V. Varadan, Multiple scattering of compressional and shear waves by fiber reinforced composite materials, J. Acoust. Soc. Am. 80 (1986) 333-339

[24] C. Aristégui and Y. Angel, Effective mass density and stiffness derived from P-wave multiple scattering, Wave Motion 44 (2007), 153-164

[25] R. M. Christensen, Mechanics of composite materials, Wiley, New-York, 1979. 
[26] P. S. Epstein and R. R. Cahart, The absorption of sound in suspensions and emulsions. I. Water fog in air, J. Acoust. Soc. Am. 25 (1953), 553565

[27] J. R. Allegra and S. A. Hawley, Attenuation of sound in suspensions and emulsions : Theory and experiments, J. Acoust. Soc. Am.51 (1972), $1545-1564$

[28] V. Tournat, V. Pagneux, D. Lafarge, and L. Jaouen, Multiple scattering of acoustic waves and porous absorbing media, Phys. Rev. E 70, 026609 (2004)

[29] J. M. Conoir and A. N. Norris, Effective wave numbers and reflection coefficients for an elastic medium containing random configurations of cylindrical scatterers, Wave Motion 47 (2010) 183-197

[30] F. Luppé, J. M. Conoir and A. N. Norris, Effective wave numbers from thermo-viscoelastic media containing random configurations of spherical scatterers, J. Acoust. Soc. Am. 131 (2012) 1113-1120

[31] D. L. Johnson, T. J. Plona and H. Kojima, Probing porous media with first and second sound. II. Acoustic properties of water-saturated porous media, J. Appl. Phys. 76(1) (1994) 115-125

[32] F. Luppé, J.M. Conoir and H. Franklin, Scattering by a fluid cylinder in a porous medium: Application to trabecular bone, J. Acoust. Soc. Am. 111 (2002) 2573-2582

[33] F. Luppé, J.-M. Conoir and S. Robert, Coherent waves in a multiply 
scattering poro-elastic medium obeying Biot's theory, W. Rand. Comp. Media 18 (2) (2008) 241-254

[34] J.-P. Sessarego, Jean Sageloli and R. Guillermin, Scattering by an elastic sphere in an elastic isotropic medium J. Acoust. Soc. Am. (1998) 104 (5) 2836-2844

[35] G. C. Gaunaurd and H. Überall, Resonance effects and the ultrasonic effective properties of particulate composites J. Acoust. Soc. Am. (1983) 74 (1) 305-313

[36] D. Brill, G. Gaunaurd and H. Überall, Resonance theory of elastic shear-wave scattering from spherical fluid obstacles in solids, J. Acoust. Soc. Am. 67 (2) (1980) 414-424

[37] M. Abramowicz and I. Stegun, Handbook of Mathematical Functions, Dover New-York, 1974

[38] Y-L Xu, Fast evaluation of the Gaunt coefficients, Mathematics of Computation, 65 (216) (1996) 1601-1612

[39] C. F. Yin and R. Truell, Scattering of a plane longitudinal wave by a spherical obstacle in an isotropically elastic solid, J. Appl. Phys. 27 (9) (1956) 1086-1097

[40] V. J. Pinfield and D. M. Forrester, Multiple scattering in random dispersions of spherical scatterers: Effects of shear-acoustic interactions, J. Acoust. Soc. Am. 141 (2017) 649-660 
[41] H. Franklin, F. Luppé and J.-M. Conoir, Multiple scattering in porous media : Comparison with water saturated double porosity media, J. Acoust. Soc. Am. 135 (5) (2014) 2513-2522

[42] J. G. Berryman, Long-wavelength propagation in composite elastic media I. Spherical inclusions, J. Acoust. Soc. Am. 68(6) (1980) 1809-1819

[43] T. J. Plona, Observation of a second bulk compressional wave in a porous medium at ultrasonic frequencies, Appl. Phys. Lett. 36(4) (1980) 259-261.

[44] Y. Bouzidi and D. R. Schmitt, Measurement of the speed and attenuation of the Biot slow wave using a large ultrasonic transmitter, J. Geophys. Res. 114 (2009) B08201 doi:10.1029/2008JB006018.

[45] R. D. Stoll and T.-K. Kan, Reflection of acoustic waves at a watersediment interface, J. Acoust. Soc. Am. 70 (1) (1981) 149-156

[46] R. J. S. Brown and J. Korringa, On the dependence of the elastic properties of a porous rock on the compressibility of the pore fluid, Geophysics (1975) 40 (4) 608-616 